\title{
Interval-valued intuitionistic multiplicative aggregation in group decision making
}

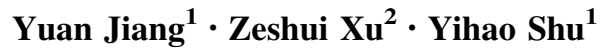

Received: 6 April 2017 / Accepted: 28 July 2017/Published online: 4 August 2017

(C) Springer International Publishing AG 2017

\begin{abstract}
Interval-valued intuitionistic multiplicative information uses an unsymmetrical scale (Saaty's 1-9) instead of the ordinary symmetrical scale to reflect our intuition which is more objective in some special situations. Recently, studies on aggregation techniques with interval-valued intuitionistic multiplicative information for group decision making (GDM) have received increasing attention, and several techniques have been proposed. However, none of them have considered the use of pseudomultiplication or Sugeno measure, and solved the GDM problems with interrelationships among input information. Since a pseudo-multiplication operator is given by weakening the axiomatic condition which is a key of fuzzy measure, and Sugeno measure is a representative non-additive measure, they are suitable for comprehensive evaluation problems which exist interactions. In this paper, we investigate interval-valued intuitionistic multiplicative aggregation operators and study the GDM problems where the preference information is given by the correlated decision makers. Based on the pseudo-multiplication, we first develop two aggregation operators (the interval-valued intuitionistic multiplicative weighted and ordered weighted averaging operators) and obtain several specific results when some specific forms are assigned. Then, for the
\end{abstract}

Zeshui Xu

xuzeshui@263.net

Yuan Jiang

jyuan0926@163.com

Yihao Shu

shuyihao0935@163.com

Unit 92919 of PLA, Ningbo, Zhejiang, China

2 Business School, Sichuan University, Chengdu, Sichuan, China situations where the input arguments have correlations or connections, we give the aggregation operator based on Choquet integral and Sugeno measure, and also study their properties. Based on the proposed aggregation operators, we present a method for GDM with interval-valued intuitionistic multiplicative preference relations, where some correlations or connections are reflected among the decision makers. Finally, we illustrate the given method with a practical example concerning the design of a satellite communication system.

Keywords Interval-valued intuitionistic multiplicative set · Group decision making - Aggregation operator

\section{Introduction}

Nowadays, decision making exists everywhere in people's daily life, and everyone faces it every day. We even cannot live without it in such a high complex society. Due to the changing and complicated socioeconomic environment, group decision making (GDM) has become a prevalent topic in the last decades (Conde and Pérez 2010; Fang et al. 2012; Jiang et al. 2013, 2015a, b, 2016; Li et al. 2017; Liang et al. 2017; Liu 2009; Orlovsky 1978; Pinar et al. 2017; Qin 2017; Ren et al. 2017; Sanchez et al. 2017; Xia et al. 2013; $\mathrm{Xu}$ 2007, 2010; $\mathrm{Xu}$ and Chen 2008; Yu 2015a, b; Yu and Shi 2015). In GDM, it is not necessary that a single decision maker should consider all important aspects of practical decision-making problems. As one of the useful tools in the process of GDM, the preference relation can express the decision makers' preferences over the given alternatives (objects or criteria), and it has quickly caught much attention and derived many desirable 
results. The preference relations can be roughly classified into two widely used forms:

1. Fuzzy preference relations (Orlovsky 1978). A fuzzy preference relation $R$ on the set $X=\left\{x_{1}, x_{2}, \ldots, x_{n}\right\}$ is represented by a complementary matrix $R=\left(r_{i j}\right)_{n \times n}$ with $0 \leq r_{i j} \leq 1, r_{i j}+r_{j i}=1, r_{i i}=0.5$, for all $i, j=$ $1,2, \ldots, n$, where $r_{i j}$ denotes the preference degree of the alternative $x_{i}$ over $x_{j}$.

2. Multiplicative preference relations (Saaty 1980). A multiplicative preference relation $P$ on the set $X=$ $\left\{x_{1}, x_{2}, \ldots, x_{n}\right\}$ is defined as a reciprocal matrix $P=$ $\left(p_{i j}\right)_{n \times n}$ under the conditions: $1 / 9 \leq p_{i j} \leq 9, p_{i j} \cdot p_{j i}=$ $1, p_{i i}=1$, for all $i, j=1,2, \ldots, n$, where $p_{i j}$ is interpreted as the ratio of the preference intensity of the alternative $x_{i}$ to $x_{j}$.

The main differences between these two kinds of preference relations are the measured scales describing information, the former is based on the 0-1 scale which is a symmetrical distribution around 0.5 (Orlovsky 1978; Xia et al. 2013), while the latter is based on Saaty's 1-9 scale which is an asymmetric distribution around 1 (Saaty 1977) (see Table 1 for more details). Since this paper focuses on the preference information measured by Saaty's 1-9 scale, we will not pay much attention on the preference information measured by the $0-1$ scale.

Sometimes, due to the time pressure and lack of information or knowledge, it is difficult for the decision makers to provide their preferences clearly. To describe the imprecision, the interval-valued fuzzy preference relations (Xu and Chen 2008) and the interval-valued multiplicative preference relations (Conde and Pérez 2010; Liu 2009; Wang and Elhag 2007) are provided to allow the decision makers to use the interval-valued numbers (Zadeh 1975) to express their preferences. It is obvious to find that the preference relations mentioned above are only characterized by a membership function describing the strength that one alternative is prior

Table 1 Saaty's 1-9 scale

\begin{tabular}{ll}
\hline $1-9$ scale & Meaning \\
\hline $1 / 9$ & Extremely not preferred \\
$1 / 7$ & Very strongly not preferred \\
$1 / 5$ & Strongly not preferred \\
$1 / 3$ & Moderately not preferred \\
1 & Equally preferred \\
3 & Moderately preferred \\
5 & Strongly preferred \\
7 & Very strongly preferred \\
9 & Extremely preferred \\
Other values between $1 / 9$ & Intermediate values used to present \\
and 9 & compromise \\
\hline
\end{tabular}

to another, but never consider the degree that one alternative is not prior to another. To deal with such cases, the intuitionistic fuzzy preference relation (Xu 2007) and the intuitionistic multiplicative preference relation (Xia et al. 2013) are defined to depict the degree that one alternative is prior to another (positive information) and the degree that one alternative is not prior to another (negative information) simultaneously. Especially, the intuitionistic multiplicative preference relation (Xia et al. 2013) can be utilized to deal with the unbalanced distribution commonly found in reallife. Generally speaking, the gap between the grades expressing good information should be larger than the one between the grades reflecting bad information. One example is the law of diminishing marginal utility in economics (Jiang et al. 2013; Xia et al. 2013). It is stated that as the amount of one factor of production increases as all other factors of production hold the same, the marginal return (extra output gained by adding an extra unit) decreases. Another example is the rain attenuation prediction for satellite communication. According to the prediction model in ITU-R (International Telecommunication Union-Radio Communication Sector) (ITU-R RPN.618-4) (1996), with improving a certain frequency, it increases more rain attenuation in the higher operating frequencies than in the lower frequencies, and causes more cost to compensate the signal outage.

However, all the positive and negative information in both the intuitionistic fuzzy and intuitionistic multiplicative preference relations need to be answered with exact values by the decision makers. Then in order to adapt the more complex characteristics of practical GDM problems, based on the interval-valued intuitionistic fuzzy sets (Atanassov and Gargov 1989), Xu and Chen extended the intuitionistic fuzzy preference relation and presented the interval-valued intuitionistic fuzzy preference relation ( $\mathrm{Xu}$ 2010); Ren et al. proposed a simplified interval-valued intuitionistic fuzzy number which was characterized by two intuitionistic fuzzy numbers (Ren et al. 2016), in which both the positive and negative information are allowed to be given by interval-valued numbers. Recently, as an extension of the intuitionistic multiplicative set (Xia et al. 2013), Jiang et al. introduced another type of set called the intervalvalued intuitionistic multiplicative set (IVIMS) (Jiang et al. 2014) and studied its operations and distance measures. Similar to the interval-valued intuitionistic fuzzy set, the IVIMS was developed in view of the cases where the positive or negative information cannot be easily given by an exact value but a probable degree range. In addition, all components of the IVIMS are measured by Saaty's 1-9 scale, not the symmetric $0-1$ scale. As an extension of the intuitionistic multiplicative preference relation, $\mathrm{Yu}$ et al. developed a new type of preference relation which is called interval-valued intuitionistic multiplicative preference fuzzy relation (Yu et al. 2013) (here we call it as the 
interval-valued intuitionistic multiplicative preference relation (IVIMPR), with regard to the homogeneity of the terms) and gave some aggregation operators based on algebraic operational laws to aggregate intuitionistic multiplicative preference information. Although many methods for decision making with interval-valued intuitionistic multiplicative information have been developed (Jiang et al. 2014; Yu 2015a, b; Yu et al. 2013), especially the aggregation techniques, there is no study on the basis of pseudo-multiplication, and no studies have considered Sugeno measure combined with Choquet integral and the situations where there exist the interrelationships among input information. Since Sugeno measure, as one type of the fuzzy measures, is a representative non-additive measure, which is useful for comprehensive evaluation problems which exist interactions in GDM. Based on the pseudo-multiplication operator, which is given by weakening the axiomatic condition, it should be effective for aggregating the interval-valued intuitionistic multiplicative information in GDM.

In this paper, based on the pseudo-multiplication, we give some different aggregation techniques which are more general to aggregate the intuitionistic multiplicative information. Besides, in order to deal with the correlated interval-valued intuitionistic multiplicative information, we apply the Choquet integral (Beliakov et al. 2007; Choquet 1953; Grabisch 1996; Yager 2004) and the Sugeno measure (also called $\lambda$ fuzzy measure) (Beliakov et al. 2007; Sugeno 1974) to propose some aggregation operators to solve the decision-making problems. Then we propose an approach to deal with the situations where all correlated decision makers provide their preference information over alternatives by using IVIMPRs.

In order to do that, the remainder of this paper is organized as follows: Sect. 2 briefly reviews some basic concepts. In Sect. 3, we mainly give some concepts related to IVIMPRs. In order to aggregate the preference information in IVIMPRs, Sect. 4 develops some interval-valued intuitionistic multiplicative aggregation operators, including the ones whose input information is non-correlated and correlated with each other. Section 5 presents an approach for GDM with IVIMPRs, and a numerical example concerning the design of a satellite communication system is given to illustrate the proposed approach by comparing it with the existing method. Section 6 ends the paper with some concluding remarks.

\section{Preliminaries}

In this section, we review some basic concepts. Let $X=$ $\left\{x_{1}, x_{2}, \ldots, x_{n}\right\}$, then an IVIMS $\tilde{D}$ on $X$ is defined as follows:
Definition 2.1 (Jiang et al. 2014) Let $X$ be fixed, an interval-valued intuitionistic multiplicative set (IVIMS) is defined as

$\tilde{D}=\left\{\left\langle x, \tilde{\rho}_{D}(x), \tilde{\sigma}_{D}(x)\right\rangle \mid x \in X\right\}$,

where $\tilde{\rho}_{D}(x) \subseteq[1 / 9,9]$ and $\tilde{\sigma}_{D}(x) \subseteq[1 / 9,9]$ are intervals satisfying $0<\sup \tilde{\rho}_{D}(x) \cdot \sup \tilde{\sigma}_{D}(x) \leq 1$, for every $x \in X$.

Especially, if $\inf \tilde{\rho}_{D}(x)=\sup \tilde{\rho}_{D}(x)$ and $\inf \tilde{\sigma}_{D}(x)=$ $\sup \tilde{\sigma}_{D}(x)$, then the IVIMS $\tilde{D}$ reduces to an intuitionistic multiplicative set. Here, the pair $\left(\tilde{\rho}_{D}(x), \tilde{\sigma}_{D}(x)\right)$ denotes an interval-valued intuitionistic multiplicative number (IVIMN). For convenience, $\left(\tilde{\rho}_{D}(x), \tilde{\sigma}_{D}(x)\right)$ can be simplified as $\left(\tilde{\rho}_{D}, \tilde{\sigma}_{D}\right)=\left(\left[\rho_{D}^{-}, \rho_{D}^{+}\right],\left[\sigma_{D}^{-}, \sigma_{D}^{+}\right]\right)$without ambiguousness, where $0<\rho_{D}^{+} \sigma_{D}^{+} \leq 1$. Let $\tilde{\tau}_{D}(x)$ be the range of uncertainty of $x$ to $X$, where $\tilde{\tau}_{D}(x) \subseteq[1,81]$, and $\tilde{\tau}_{D}(x)$ can be simplified as $\tilde{\tau}_{D}=\left[\tau_{D}^{-}, \tau_{D}^{+}\right]$with the conditions $\tau_{D}^{-}=$ $1 / \rho_{D}^{+} \sigma_{D}^{+}$and $\tau_{D}^{+}=1 / \rho_{D}^{-} \sigma_{D}^{-}$.

Definition 2.2 (Yu et al. 2013) Let $\tilde{a}, \tilde{a}_{1}$, and $\tilde{a}_{2}$ be three IVIMNs, $s(\tilde{a})=\sqrt{\rho_{a}^{+} \rho_{a}^{-} / \sigma_{a}^{-} \sigma_{a}^{+}}$be the score function of $\tilde{a}$, and $r(\tilde{a})=\sqrt{\rho_{a}^{-} \rho_{a}^{+} \sigma_{a}^{-} \sigma_{a}^{+}}$be the accuracy function of $\tilde{a}$, then

1. If $s\left(\tilde{a}_{1}\right)>s\left(\tilde{a}_{2}\right)$, then $\tilde{a}_{1}>\tilde{a}_{2}$;

2. If $s\left(\tilde{a}_{1}\right)=s\left(\tilde{a}_{2}\right)$, then

(i) If $r\left(\tilde{a}_{1}\right)>r\left(\tilde{a}_{2}\right)$, then $\tilde{a}_{1}>\tilde{a}_{2}$;

(ii) If $r\left(\tilde{a}_{1}\right)=r\left(\tilde{a}_{2}\right)$, then $\tilde{a}_{1}=\tilde{a}_{2}$.

Pseudo-multiplications are a special kind of associative binary relations satisfying a series of properties. It is given by weakening the axiomatic condition (Benvenuti and Mesiar 2004; Sugeno 1974; Sugeno and Murofushi 1987). Based on the pseudo-multiplication $\odot$ which is defined as: $x \odot y=g^{-1}(g(x) g(y))$, where $g$ is a strictly decreasing function such that $g(t):(0, \infty) \rightarrow(0, \infty)$ (Pap and Ralević 1998), some interval-valued intuitionistic multiplicative operations about IVIMNs were also given (Jiang et al. 2014):

Definition 2.3 (Jiang et al. 2014) Let $\tilde{a}=\left(\tilde{\rho}_{a}, \tilde{\sigma}_{a}\right)=$ $\left(\left[\rho_{a}^{-}, \rho_{a}^{+}\right],\left[\sigma_{a}^{-}, \sigma_{a}^{+}\right]\right)$and $\tilde{a}_{i}=\left(\tilde{\rho}_{a_{i}}, \tilde{\sigma}_{a_{i}}\right)(i=1,2)$ be three IVIMNs, and $\lambda>0$, then we have

1. $\tilde{a}_{1}+\tilde{a}_{2}=\left(\left[h^{-1}\left(h\left(\rho_{a_{1}}^{-}\right) h\left(\rho_{a_{2}}^{-}\right)\right), h^{-1}\left(h\left(\rho_{a_{1}}^{+}\right) h\left(\rho_{a_{2}}^{+}\right)\right)\right]\right.$, $\left.\left[g^{-1}\left(g\left(\sigma_{a_{1}}^{-}\right) g\left(\sigma_{a_{2}}^{-}\right)\right), g^{-1}\left(g\left(\sigma_{a_{1}}^{+}\right) g\left(\sigma_{a_{2}}^{+}\right)\right)\right]\right)$;

2. $\tilde{a}_{1} \cdot \tilde{a}_{2}=\left(\left[g^{-1}\left(g\left(\rho_{a_{1}}^{-}\right) g\left(\rho_{a_{2}}^{-}\right)\right), g^{-1}\left(g\left(\rho_{a_{1}}^{+}\right) g\left(\rho_{a_{2}}^{+}\right)\right)\right]\right.$, $\left.\left[h^{-1}\left(h\left(\sigma_{a_{1}}^{-}\right) h\left(\sigma_{a_{2}}^{-}\right)\right), g^{-1}\left(h\left(\sigma_{a_{1}}^{+}\right) h\left(\sigma_{a_{2}}^{+}\right)\right)\right]\right)$;

3. $\lambda \tilde{a}=\left(\left[h^{-1}\left(\left(h\left(\rho_{a}^{-}\right)\right)^{\lambda}\right), h^{-1}\left(\left(h\left(\rho_{a}^{+}\right)\right)^{\lambda}\right)\right]\right.$, $\left.\left[g^{-1}\left(\left(g\left(\sigma_{a}^{-}\right)\right)^{\lambda}\right), g^{-1}\left(\left(g\left(\sigma_{a}^{+}\right)\right)^{\lambda}\right)\right]\right)$; 
4. $\tilde{a}^{\lambda}=\left(\left[g^{-1}\left(\left(g\left(\rho_{a}^{-}\right)\right)^{\lambda}\right), g^{-1}\left(\left(g\left(\rho_{a}^{+}\right)\right)^{\lambda}\right)\right]\right.$, $\left.\left[h^{-1}\left(\left(h\left(\sigma_{a}^{-}\right)\right)^{\lambda}\right), h^{-1}\left(\left(h\left(\sigma_{a}^{+}\right)\right)^{\lambda}\right)\right]\right)$,

where $g$ is a strictly decreasing function such that $g(t):(0, \infty) \rightarrow(0, \infty)$, and $h(t)=g(1 / t)$.

Furthermore, the following relations and operations are also proven to be valid in Jiang et al. (2014):

Theorem 2.1 (Jiang et al. 2014) Let $\tilde{a}=\left(\tilde{\rho}_{a}, \tilde{\sigma}_{a}\right)=$ $\left(\left[\rho_{a}^{-}, \rho_{a}^{+}\right],\left[\sigma_{a}^{-}, \sigma_{a}^{+}\right]\right)$and $\tilde{a}_{i}=\left(\tilde{\rho}_{a_{i}}, \tilde{\sigma}_{a_{i}}\right) \quad(i=1,2)$ be three IVIMNs, and $\lambda, \lambda_{1}, \lambda_{2}>0$, then we have

1. $\tilde{a}_{1}+\tilde{a}_{2}=\tilde{a}_{2}+\tilde{a}_{1}$

2. $\quad \tilde{a}_{1} \cdot \tilde{a}_{2}=\tilde{a}_{2} \cdot \tilde{a}_{1}$

3. $\lambda\left(\tilde{a}_{1}+\tilde{a}_{2}\right)=\lambda \tilde{a}_{1}+\lambda \tilde{a}_{2}$;

4. $\left(\tilde{a}_{1} \cdot \tilde{a}_{2}\right)^{\lambda}=\tilde{a}_{1}^{\lambda} \cdot \tilde{a}_{2}^{\lambda}$;

5. $\lambda_{1} \tilde{a}+\lambda_{2} \tilde{a}=\left(\lambda_{1}+\lambda_{2}\right) \tilde{a}$;

6. $\tilde{a}^{\lambda_{1}} \cdot \tilde{a}^{\lambda_{2}}=\tilde{a}^{\lambda_{1}+\lambda_{2}}$.

Let $\tilde{a}^{C}=\left(\tilde{\sigma}_{a}, \tilde{\rho}_{a}\right)$ denote the complement of $\tilde{a}$, then

7. $\left(\tilde{a}^{C}\right)^{\lambda}=(\lambda \tilde{a})^{C}$;

8. $\lambda\left(\tilde{a}^{C}\right)=\left(\tilde{a}^{\lambda}\right)^{C}$

9. $\tilde{a}_{1}^{C}+\tilde{a}_{2}^{C}=\left(\tilde{a}_{1} \cdot \tilde{a}_{2}\right)^{C}$;

10. $\tilde{a}_{1}^{C} \cdot \tilde{a}_{2}^{C}=\left(\tilde{a}_{1}+\tilde{a}_{2}\right)^{C}$.

\section{Interval-valued intuitionistic multiplicative preference relations}

Preference relation is one of the convenient techniques to express the decision makers' preferences over alternatives, by which the ranking of alternatives can be obtained. For a set of alternatives $X=\left\{x_{1}, x_{2}, \ldots, x_{n}\right\}$, the decision makers compare each pair of alternatives and construct judgment matrixes, respectively. If every component in the judgment matrixes is an IVIMN, then the concept of the IVIMPR can be given. For better and further studies, it is necessary to give a precise definition based on $\mathrm{Yu}$ et al. (2013) as follows:

Definition 3.1 Let $X=\left\{x_{1}, x_{2}, \ldots, x_{n}\right\}$ be $n$ alternatives, then the interval-valued intuitionistic multiplicative preference relation is defined as $\tilde{A}=\left(\tilde{a}_{i j}\right)_{n \times n}$, where $\tilde{a}_{i j}=$ $\left(\tilde{\rho}_{i j}, \tilde{\sigma}_{i j}\right)$ is an IVIMN, for all $i, j=1,2, \ldots, n, \tilde{\rho}_{i j}$ indicates the preference degree of the alternative $x_{i}$ over $x_{j}$, while $\tilde{\sigma}_{i j}$ indicates the preference degree of the alternative $x_{j}$ over $x_{i}$, and both of them should satisfy the following condition: $\tilde{\rho}_{i j}=\tilde{\sigma}_{j i}, \quad \tilde{\sigma}_{i j}=\tilde{\rho}_{j i}$,

$0<\sup \tilde{\rho}_{i j} \cdot \sup \tilde{\sigma}_{i j} \leq 1, \quad \tilde{\rho}_{i j}, \tilde{\sigma}_{i j} \subseteq[1 / 9,9]$

We can easily get $\tilde{\rho}_{i i}=\tilde{\sigma}_{i i}=([1,1],[1,1])$ for any IVIMPR. Moreover, if $\tau_{i j}^{-}=1 / \rho_{i j}^{+} \sigma_{i j}^{+}$and $\tau_{i j}^{+}=1 / \rho_{i j}^{-} \sigma_{i j}^{-}$, then $\tilde{\tau}_{i j}=\left[\tau_{i j}^{-}, \tau_{i j}^{+}\right]$denotes the uncertainty degree of $x_{i}$ over $x_{j}$, where $\tilde{\tau}_{i j} \subseteq[1,81]$.

Especially, if $\rho_{i j}^{-}=\rho_{i j}^{+}, \sigma_{i j}^{-}=\sigma_{i j}^{+}$, for all $i, j$, then the IVIMPR $\tilde{A}$ reduces to an intuitionistic multiplicative preference relation, besides, if $\tau_{i j}^{-}=\tau_{i j}^{+}=1$, then the IVIMPR $\tilde{A}$ reduces to a traditional multiplicative preference relation.

It is noted that: (a) Compared with the intuitionistic multiplicative preference relations (Xia et al. 2013), IVIMPRs allow the decision makers to give their preferences over alternatives by using probable degree ranges, not exact values necessarily which are sometimes difficult to be given due to the time pressure and lack of information or knowledge. (b) Compared with the interval-valued multiplicative preference relations (Conde and Pérez 2010; Liu 2009; Wang and Elhag 2007), the IVIMPRs contain the decision makers' preferences including the positive and negative information, without ignoring the degree that one alternative is not prior to another. (c) Compared with the intervalvalued fuzzy and intuitionistic fuzzy preference relations (Xu 2007; Xu and Chen 2008), the IVIMPRs permit the decision makers to express their preferences over alternatives by an asymmetric measured scale (Saaty's 1-9 scale) which can be used to model the unbalanced distribution (Xia et al. 2013) commonly found in real-life, but not necessarily symmetrical scale.

In what follows, we give some corresponding concepts of the IVIMPR:

Definition 3.2 Let $\tilde{A}=\left(\tilde{a}_{i j}\right)_{n \times n}$ be an IVIMPR, where $\tilde{a}_{i j}=\left(\tilde{\rho}_{i j}, \tilde{\sigma}_{i j}\right)$, then $\tilde{A}^{T}=\left(\tilde{a}_{i j}^{T}\right)_{n \times n}=\left(\tilde{a}_{j i}\right)_{n \times n}$ is called the transposition of $\tilde{A}$, where $\tilde{a}_{j i}=\left(\tilde{\rho}_{j i}, \tilde{\sigma}_{j i}\right)$, for all $i, j=1,2, \ldots, n$.

Definition 3.3 Let $\tilde{A}=\left(\tilde{a}_{i j}\right)_{n \times n}$ be an IVIMPR, where $\tilde{a}_{i j}=\left(\tilde{\rho}_{i j}, \tilde{\sigma}_{i j}\right)$, then $S(\tilde{A})=\left(s_{i j}\right)_{n \times n}$ is called the score matrix of $\tilde{A}$, where $s_{i j}$ is derived by the score function, such that

$s_{i j}=\sqrt{\rho_{i j}^{+} \rho_{i j}^{-} / \sigma_{i j}^{-} \sigma_{i j}^{+}}, \quad i, j=1,2, \ldots, n$. 
Definition 3.4 Let $\tilde{A}=\left(\tilde{a}_{i j}\right)_{n \times n}$ be an IVIMPR, where $\tilde{a}_{i j}=\left(\tilde{\rho}_{i j}, \tilde{\sigma}_{i j}\right)$, then $R(\tilde{A})=\left(r_{i j}\right)_{n \times n}$ is called the accuracy matrix of $\tilde{A}$, where $r_{i j}$ is derived by the accuracy function, such that

$r_{i j}=\sqrt{\rho_{i j}^{-} \rho_{i j}^{+} \sigma_{i j}^{-} \sigma_{i j}^{+}}, \quad(i, j=1,2, \ldots, n)$.

Score and accuracy functions are useful tools in the process of matrix calculation. Usually, they are used for solving the decision-making problems, especially GDM.

Theorem 3.1 Let $\tilde{A}^{T}=\left(\tilde{a}_{i j}^{T}\right)_{n \times n}$ be the transposition of the IVIMPR $\tilde{A}$, then we have $\tilde{A}^{T}=\left(\tilde{a}_{i j}^{C}\right)_{n \times n}$, where $\tilde{a}_{i j}^{C}=\left(\tilde{\sigma}_{i j}, \tilde{\rho}_{i j}\right)$, for all $i, j=1,2, \ldots, n$.

Proof From Eq. (2), we have $\tilde{\rho}_{i j}=\tilde{\sigma}_{j i}$ and $\tilde{\sigma}_{i j}=\tilde{\rho}_{j i}$ then $\tilde{A}^{T}=\left(\tilde{a}_{j i}\right)_{n \times n}=\left(\tilde{\rho}_{j i}, \tilde{\sigma}_{j i}\right)_{n \times n}=\left(\tilde{\sigma}_{i j}, \tilde{\rho}_{i j}\right)_{n \times n}=\left(\tilde{a}_{i j}^{C}\right)_{n \times n}$.

Obviously, the transposition of the symmetric IVIMPR equals to itself. Next, we continue to study the score and accuracy matrices.

Theorem 3.2 Let $\tilde{A}=\left(\tilde{a}_{i j}\right)_{n \times n}$ be an IVIMPR, then

$S^{T}(\tilde{A})=S\left(\tilde{A}^{T}\right), \quad S(\tilde{A}) \circ S\left(\tilde{A}^{T}\right)=Z_{n \times n}$

for all $i, j=1,2, \ldots, n$, where "o" denotes the Hadamard product and $Z$ indicates a matrix whose elements are all equal to 1.

Proof Due to that $\tilde{A}^{T}=\left(\tilde{a}_{j i}\right)_{n \times n}$ is the transposition of $\tilde{A}$, $S(\tilde{A})=\left(s_{i j}\right)_{n \times n}$ is the score matrix of $\tilde{A}, S^{T}(\tilde{A})$ is the transposition of $S(\tilde{A})$, and $S\left(\tilde{A}^{T}\right)$ is the score matrix if $\tilde{A}^{T}$, we have

$$
\begin{aligned}
& \tilde{A}=\left(\left[\rho_{i j}^{-}, \rho_{i j}^{+}\right],\left[\sigma_{i j}^{-}, \sigma_{i j}^{+}\right]\right)_{n \times n}, \\
& \tilde{A}^{T}=\left(\left[\rho_{j i}^{-}, \rho_{j i}^{+}\right],\left[\sigma_{j i}^{-}, \sigma_{j i}^{+}\right]\right)_{n \times n}, \\
& S^{T}(\tilde{A})=\left(\sqrt{\rho_{i j}^{+} \rho_{i j}^{-} / \sigma_{i j}^{-} \sigma_{i j}^{+}}\right)_{n \times n}^{T}=\left(\sqrt{\rho_{j i}^{+} \rho_{j i}^{-} / \sigma_{j i}^{-} \sigma_{j i}^{+}}\right)_{n \times n} \\
& =S\left(\tilde{A}^{T}\right) .
\end{aligned}
$$

Then based on Theorem 3.1, $\tilde{A}^{T}=\left(\tilde{a}_{i j}^{C}\right)_{n \times n}$, and thus, we get

$$
\begin{aligned}
S(\tilde{A}) \circ S\left(\tilde{A}^{T}\right) & =\left(\sqrt{\rho_{i j}^{+} \rho_{i j}^{-} / \sigma_{i j}^{-} \sigma_{i j}^{+}}\right)_{n \times n} \circ\left(\sqrt{\rho_{j i}^{+} \rho_{j i}^{-} / \sigma_{j i}^{-} \sigma_{j i}^{+}}\right)_{n \times n} \\
& =\left(\sqrt{\rho_{i j}^{+} \rho_{i j}^{-} / \sigma_{i j}^{-} \sigma_{i j}^{+}} \cdot \sqrt{\sigma_{i j}^{+} \sigma_{i j}^{-} / \rho_{i j}^{-} \rho_{i j}^{+}}\right)_{n \times n}=Z_{n \times n} .
\end{aligned}
$$

Theorem 3.3 Let $\tilde{A}=\left(\tilde{a}_{i j}\right)_{n \times n}$ be an IVIMPR, then

$R^{T}(\tilde{A})=R(\tilde{A}), \quad R(\tilde{A})=R\left(\tilde{A}^{T}\right)$

which implies that $R$ is symmetric, for all $i, j=1,2, \ldots, n$.

Proof Since $R(\tilde{A})=\left(r_{i j}\right)_{n \times n}$ is the accuracy matrix of $\tilde{A}$, $R^{T}(\tilde{A})$ is the transposition of $R(\tilde{A})$ and $R\left(\tilde{A}^{T}\right)$ is the score matrix if $\tilde{A}^{T}$, then

$R(\tilde{A})=\left(\sqrt{\rho_{i j}^{-} \rho_{i j}^{+} \sigma_{i j}^{-} \sigma_{i j}^{+}}\right)_{n \times n}$

and from Eq. (2), we have

$$
\begin{aligned}
R^{T}(\tilde{A}) & =\left(\sqrt{\rho_{\overline{j i}}^{-} \rho_{j i}^{+} \sigma_{j i}^{-} \sigma_{j i}^{+}}\right)_{n \times n}=\left(\sqrt{\sigma_{i j}^{-} \sigma_{i j}^{+} \rho_{i j}^{-} \rho_{i j}^{+}}\right)_{n \times n} \\
& =R(\tilde{A})
\end{aligned}
$$

and then according to Theorem 3.1, we have

$R\left(\tilde{A}^{T}\right)=\left(\sqrt{\sigma_{i j}^{-} \sigma_{i j}^{+} \rho_{i j}^{-} \rho_{i j}^{+}}\right)_{n \times n}=R(\tilde{A})$.

Next, we use an example to illustrate the IVIMPR:

Example 1 Let $\tilde{A}=\left(\tilde{a}_{i j}\right)_{n \times n}(i, j=1,2, \ldots, n)$ be an IVIMPR such that

$$
\tilde{A}=\left(\begin{array}{ccc}
([1,1],[1,1]) & ([3,5],[1 / 6,1 / 5]) & ([2,3],[1 / 8,1 / 6]) \\
([1 / 6,1 / 5],[3,5]) & ([1,1],[1,1]) & ([4,6],[1 / 8,1 / 7]) \\
([1 / 8,1 / 6],[2,3]) & ([1 / 8,1 / 7],[4,6]) & ([1,1],[1,1])
\end{array}\right)_{3 \times 3}
$$

Then based on Definition 3.2, the transposition of $\tilde{A}$ is $\tilde{A}^{T}=\left(\tilde{a}_{j i}\right)_{n \times n}$ :

$$
\tilde{A}^{T}=\left(\begin{array}{ccc}
([1,1],[1,1]) & ([1 / 6,1 / 5],[3,5]) & ([1 / 8,1 / 6],[2,3]) \\
([3,5],[1 / 6,1 / 5]) & ([1,1],[1,1]) & ([1 / 8,1 / 7],[4,6]) \\
([2,3],[1 / 8,1 / 6]) & ([4,6],[1 / 8,1 / 7]) & ([1,1],[1,1])
\end{array}\right)_{3 \times 3}
$$

and based on Definition 3.3, the score matrixes of $\tilde{A}$ and $\tilde{A}^{T}$ are as follows:

$$
\begin{aligned}
& S(\tilde{A})=\left(\begin{array}{ccc}
1 & 0.0471 & 0.0589 \\
21.2132 & 1 & 0.0273 \\
16.9706 & 36.6606 & 1
\end{array}\right)_{3 \times 3}, \\
& S\left(\tilde{A}^{T}\right)=\left(\begin{array}{ccc}
1 & 21.2132 & 16.9706 \\
0.0471 & 1 & 36.6606 \\
0.0589 & 0.0273 & 1
\end{array}\right)_{3 \times 3} .
\end{aligned}
$$

We can easily find $S^{T}(\tilde{A})=S\left(\tilde{A}^{T}\right)$, and 
$S(\tilde{A}) \circ S\left(\tilde{A}^{T}\right)=\left(\begin{array}{lll}1 & 1 & 1 \\ 1 & 1 & 1 \\ 1 & 1 & 1\end{array}\right)_{3 \times 3}$

Based on Definition 3.4, we get the accuracy matrixes of $\tilde{A}$ and $\tilde{A}^{T}$ :

$$
\begin{aligned}
R(\tilde{A}) & =\left(\begin{array}{ccc}
1 & 0.7071 & 0.3536 \\
0.7071 & 1 & 0.6547 \\
0.3536 & 0.6547 & 1
\end{array}\right)_{3 \times 3} \\
& =R\left(\tilde{A}^{T}\right)=R^{T}(\tilde{A}) .
\end{aligned}
$$

\section{Interval-valued intuitionistic multiplicative aggregation operators}

To aggregate the interval-valued intuitionistic multiplicative information, in what follows, we develop some interval-valued intuitionistic multiplicative aggregation operators including the ones whose input information is non-correlated or correlated.

\subsection{Some aggregation operators with interval- valued intuitionistic multiplicative operations}

In this section, we mainly apply the operational laws reviewed in Sect. 2 (Definition 2.3 and Theorem 2.1) to aggregate the interval-valued intuitionistic multiplicative information:

Definition 4.1 (Yu et al. 2013) Let $\tilde{a}_{i}=\left(\tilde{\rho}_{a_{i}}, \tilde{\sigma}_{a_{i}}\right)(i=1,2, \ldots, n)$ be a collection of IVIMNs, and $\omega=\left(\omega_{1}, \omega_{2}, \ldots, \omega_{n}\right)^{T}$ be the weight vector of $\tilde{a}_{i}$, where $\omega_{i}$ indicates the importance degree of $\tilde{a}_{i}$ satisfying $\omega_{i} \in[0,1]$ and $\sum_{i=1}^{n} \omega_{i}=1$, if

$\operatorname{IVIMWA}\left(\tilde{a}_{1}, \tilde{a}_{2}, \ldots, \tilde{a}_{n}\right)=\sum_{i=1}^{n} \omega_{i} \tilde{a}_{i}$

then the function IVIMWA is called the interval-valued intuitionistic multiplicative weighted averaging (IVIMWA) operator. Here, we call the IMIFWA operator in (Yu et al. 2013) as the IVIMWA operator with regard to the homogeneity of the terms.

Especially, if $\omega=(1 / n, 1 / n, \ldots, 1 / n)^{T}$, then the IVIMWA operator reduces to the interval-valued intuitionistic multiplicative averaging (IVIMA) operator:

$\operatorname{IVIMA}\left(\tilde{a}_{1}, \tilde{a}_{2}, \ldots, \tilde{a}_{n}\right)=\frac{1}{n} \sum_{i=1}^{n} \tilde{a}_{i}$.

Theorem 4.1 Let $\tilde{a}_{i}=\left(\tilde{\rho}_{a_{i}}, \tilde{\sigma}_{a_{i}}\right)(i=1,2, \ldots, n)$ be $a$ collection of IVIMNs, and $\omega=\left(\omega_{1}, \omega_{2}, \ldots, \omega_{n}\right)^{T}$ be the weight vector of them, where $\omega_{i}$ indicates the importance degree of $\tilde{a}_{i}$ satisfying $\omega_{i} \in[0,1]$ and $\sum_{i=1}^{n} \omega_{i}=1$, then the aggregated value by using the IVIMWA operator is also an IVIMN, and

$$
\begin{aligned}
& \operatorname{IVIMWA}\left(\tilde{a}_{1}, \tilde{a}_{2}, \ldots, \tilde{a}_{n}\right) \\
& \quad=\left(\left[h^{-1}\left(\prod_{i=1}^{n}\left(h\left(\rho_{a_{i}}^{-}\right)\right)^{\omega_{i}}\right), \quad h^{-1}\left(\prod_{i=1}^{n}\left(h\left(\rho_{a_{i}}^{+}\right)\right)^{\omega_{i}}\right)\right],\right. \\
& \left.\left[g^{-1}\left(\prod_{i=1}^{n}\left(g\left(\sigma_{a_{i}}^{-}\right)\right)^{\omega_{i}}\right), \quad g^{-1}\left(\prod_{i=1}^{n}\left(g\left(\sigma_{a_{i}}^{+}\right)\right)^{\omega_{i}}\right)\right]\right) .
\end{aligned}
$$

Proof By using the mathematical induction on $n$, for $n=2$, we have

$$
\begin{aligned}
& \operatorname{IVIMWA}\left(\tilde{a}_{1}, \tilde{a}_{2}\right)=\sum_{i=1}^{2} \omega_{i} \tilde{a}_{i}=\omega_{1} \tilde{a}_{1}+\omega_{2} \tilde{a}_{2} \\
& =\left(\left[h^{-1}\left(h\left(h^{-1}\left(\left(h\left(\rho_{a_{1}}^{-}\right)\right)^{\omega_{1}}\right)\right) \cdot h\left(h^{-1}\left(\left(h\left(\rho_{a_{2}}^{-}\right)\right)^{\omega_{2}}\right)\right)\right), h^{-1}\left(h\left(h^{-1}\left(\left(h\left(\rho_{a_{1}}^{+}\right)\right)^{\omega_{1}}\right)\right) \cdot h\left(h^{-1}\left(\left(h\left(\rho_{a_{2}}^{+}\right)\right)^{\omega_{2}}\right)\right)\right)\right],\right. \\
& \left.\left[g^{-1}\left(g\left(g^{-1}\left(\left(g\left(\sigma_{a_{1}}^{-}\right)\right)^{\omega_{1}}\right)\right) \cdot g\left(g^{-1}\left(\left(g\left(\sigma_{a_{2}}^{-}\right)\right)^{\omega_{2}}\right)\right)\right), h^{-1}\left(h\left(h^{-1}\left(\left(g\left(\sigma_{a_{1}}^{+}\right)\right)^{\omega_{1}}\right)\right) \cdot h\left(h^{-1}\left(\left(g\left(\sigma_{a_{2}}^{+}\right)\right)^{\omega_{2}}\right)\right)\right)\right]\right) \\
& =\left(\left[h^{-1}\left(\left(h\left(\rho_{a_{1}}^{-}\right)\right)^{\omega_{1}} \cdot\left(h\left(\rho_{a_{2}}^{-}\right)\right)^{\omega_{2}}\right), h^{-1}\left(\left(h\left(\rho_{a_{1}}^{+}\right)\right)^{\omega_{1}} \cdot\left(h\left(\rho_{a_{2}}^{+}\right)\right)^{\omega_{2}}\right)\right],\right. \\
& \left.\left[g^{-1}\left(\left(g\left(\sigma_{a_{1}}^{-}\right)\right)^{\omega_{1}} \cdot\left(g\left(\sigma_{a_{2}}^{-}\right)\right)^{\omega_{2}}\right), g^{-1}\left(\left(g\left(\sigma_{a_{1}}^{+}\right)\right)^{\omega_{1}} \cdot\left(g\left(\sigma_{a_{2}}^{+}\right)\right)^{\omega_{2}}\right)\right]\right) .
\end{aligned}
$$


Suppose that Eq. (9) holds for $n=k$, that is

$$
\begin{aligned}
& \operatorname{IVIMWA}\left(\tilde{a}_{1}, \tilde{a}_{2}, \ldots, \tilde{a}_{k}\right)=\sum_{i=1}^{k} \omega_{i} \tilde{a}_{i}=\omega_{1} \tilde{a}_{1}+\omega_{2} \tilde{a}_{2}+\cdots+\omega_{k} \tilde{a}_{k} \\
& =\left(\left[h^{-1}\left(\prod_{i=1}^{k}\left(h\left(\rho_{a_{i}}^{-}\right)\right)^{\omega_{i}}\right), h^{-1}\left(\prod_{i=1}^{k}\left(h\left(\rho_{a_{i}}^{+}\right)\right)^{\omega_{i}}\right)\right],\right. \\
& \left.\left[g^{-1}\left(\prod_{i=1}^{k}\left(g\left(\sigma_{a_{i}}^{-}\right)\right)^{\omega_{i}}\right), g^{-1}\left(\prod_{i=1}^{k}\left(g\left(\sigma_{a_{i}}^{+}\right)\right)^{\omega_{i}}\right)\right]\right),
\end{aligned}
$$

then we have
Then, we get

$$
\begin{aligned}
& {\left[h^{-1}\left(\prod_{i=1}^{n}\left(h\left(\rho_{a_{i}}^{-}\right)\right)^{\omega_{i}}\right), h^{-1}\left(\prod_{i=1}^{n}\left(h\left(\rho_{a_{i}}^{+}\right)\right)^{\omega_{i}}\right)\right] \subseteq[1 / 9,9]} \\
& \text { and }\left[g^{-1}\left(\prod_{i=1}^{n}\left(g\left(\sigma_{a_{i}}^{-}\right)\right)^{\omega_{i}}\right), g^{-1}\left(\prod_{i=1}^{n}\left(g\left(\sigma_{a_{i}}^{+}\right)\right)^{\omega_{i}}\right)\right] \subseteq[1 / 9,9]
\end{aligned}
$$

and

$\operatorname{IVIMWA}\left(\tilde{a}_{1}, \tilde{a}_{2}, \ldots, \tilde{a}_{k}, \tilde{a}_{k+1}\right)=\sum_{i=1}^{k} \omega_{i} \tilde{a}_{i}+\omega_{k+1} \tilde{a}_{k+1}$

$$
\begin{aligned}
& =\left(\left[h^{-1}\left(\prod_{i=1}^{k}\left(h\left(\rho_{a_{i}}^{-}\right)\right)^{\omega_{i}}\right), h^{-1}\left(\prod_{i=1}^{k}\left(h\left(\rho_{a_{i}}^{+}\right)\right)^{\omega_{i}}\right)\right],\left[g^{-1}\left(\prod_{i=1}^{k}\left(g\left(\sigma_{a_{i}}^{-}\right)\right)^{\omega_{i}}\right), g^{-1}\left(\prod_{i=1}^{k}\left(g\left(\sigma_{a_{i}}^{+}\right)\right)^{\omega_{i}}\right)\right]\right) \\
& +\left(\left[h^{-1}\left(\left(h\left(\rho_{a_{k+1}}^{-}\right)\right)^{\omega_{k+1}}\right), h^{-1}\left(\left(h\left(\rho_{a_{k+1}}^{+}\right)\right)^{\omega_{k+1}}\right)\right],\left[g^{-1}\left(\left(g\left(\sigma_{a_{k+1}}^{-}\right)\right)^{\omega_{k+1}}\right), g^{-1}\left(\left(g\left(\sigma_{a_{k+1}}^{+}\right)\right)^{\omega_{k+1}}\right)\right]\right) \\
& =\left(\left[h^{-1}\left(h\left(h^{-1}\left(\prod_{i=1}^{k}\left(h\left(\rho_{a_{i}}^{-}\right)\right)^{\omega_{i}}\right)\right) h\left(h^{-1}\left(\left(h\left(\rho_{a_{k+1}}^{-}\right)\right)^{\omega_{k+1}}\right)\right)\right), h^{-1}\left(h\left(h^{-1}\left(\prod_{i=1}^{k}\left(h\left(\rho_{a_{i}}^{+}\right)\right)^{\omega_{i}}\right)\right) h\left(h^{-1}\left(\left(h\left(\rho_{a_{k+1}}^{+}\right)\right)^{\omega_{k+1}}\right)\right)\right)\right],\right. \\
& \left.\left[g^{-1}\left(g\left(g^{-1}\left(\prod_{i=1}^{k}\left(g\left(\sigma_{a_{i}}^{-}\right)\right)^{\omega_{i}}\right)\right) \cdot g\left(g^{-1}\left(\left(g\left(\sigma_{a_{k+1}}^{-}\right)\right)^{\omega_{k+1}}\right)\right)\right), g^{-1}\left(g\left(g^{-1}\left(\prod_{i=1}^{k}\left(g\left(\sigma_{a_{i}}^{+}\right)\right)^{\omega_{i}}\right)\right) \cdot g\left(g^{-1}\left(\left(g\left(\sigma_{a_{k+1}}^{+}\right)\right)^{\omega_{k+1}}\right)\right)\right)\right]\right) \\
& =\left(\left[h^{-1}\left(\prod_{i=1}^{k}\left(h\left(\rho_{a_{i}}^{-}\right)\right)^{\omega_{i}} \cdot\left(h\left(\rho_{a_{k+1}}^{-}\right)\right)^{\omega_{k+1}}\right), h^{-1}\left(\prod_{i=1}^{k}\left(h\left(\rho_{a_{i}}^{+}\right)\right)^{\omega_{i}} \cdot\left(h\left(\rho_{a_{k+1}}^{+}\right)\right)^{\omega_{k+1}}\right)\right],\right. \\
& \left.\left[g^{-1}\left(\prod_{i=1}^{k}\left(g\left(\sigma_{a_{i}}^{-}\right)\right)^{\omega_{i}} \cdot\left(g\left(\sigma_{a_{k+1}}^{-}\right)\right)^{\omega_{k+1}}\right), g^{-1}\left(\prod_{i=1}^{k} g\left(\left(\sigma_{a_{i}}^{+}\right)\right)^{\omega_{i}} \cdot\left(g\left(\sigma_{a_{k+1}}^{+}\right)\right)^{\omega_{k+1}}\right)\right]\right) \\
& =\left(\left[h^{-1}\left(\prod_{i=1}^{k+1}\left(h\left(\rho_{a_{i}}^{-}\right)\right)^{\omega_{i}}\right), h^{-1}\left(\prod_{i=1}^{k+1}\left(h\left(\rho_{a_{i}}^{+}\right)\right)^{\omega_{i}}\right)\right],\left[g^{-1}\left(\prod_{i=1}^{k+1}\left(g\left(\sigma_{a_{i}}^{-}\right)\right)^{\omega_{i}}\right), g^{-1}\left(\prod_{i=1}^{k+1}\left(g\left(\sigma_{a_{i}}^{+}\right)\right)^{\omega_{i}}\right)\right]\right),
\end{aligned}
$$

i.e., Eq. (9) holds for $n=k+1$. Thus, Eq. (9) holds for all $n$.

In addition, we have known that $h(t)=g(1 / t)$, and $g$ is a strictly decreasing function such that $g(t):(0,+\infty) \rightarrow$ $(0,+\infty)$; therefore, $h(t)$ is a strictly increasing function implying that

$$
\begin{aligned}
0<h^{-1}\left(\prod_{i=1}^{n}\left(h\left(\rho_{a_{i}}^{-}\right)\right)^{\omega_{i}}\right), & h^{-1}\left(\prod_{i=1}^{n}\left(h\left(\rho_{a_{i}}^{+}\right)\right)^{\omega_{i}}\right), \\
& g^{-1}\left(\prod_{i=1}^{n}\left(g\left(\sigma_{a_{i}}^{-}\right)\right)^{\omega_{i}}\right), \quad g^{-1}\left(\prod_{i=1}^{n}\left(g\left(\sigma_{a_{i}}^{+}\right)\right)^{\omega_{i}}\right)<+\infty .
\end{aligned}
$$

Moreover,

$\frac{1}{9}=\frac{1}{9} \sum_{i=1}^{n} \omega_{i}=h^{-1}\left(\prod_{i=1}^{n}(h(1 / 9))^{\omega_{i}}\right) \leq h^{-1}\left(\prod_{i=1}^{n}\left(h\left(\rho_{a_{i}}^{-}\right)\right)^{\omega_{i}}\right)$

$\leq h^{-1}\left(\prod_{i=1}^{n}\left(h\left(\rho_{a_{i}}^{+}\right)\right)^{\omega_{i}}\right) \leq h^{-1}\left(\prod_{i=1}^{n}(h(9))^{\omega_{i}}\right)=9 \sum_{i=1}^{n} \omega_{i}=9$

$\frac{1}{9}=\frac{1}{9} \sum_{i=1}^{n} \omega_{i}=g^{-1}\left(\prod_{i=1}^{n} g(1 / 9)^{\omega_{i}}\right) \leq g^{-1}\left(\prod_{i=1}^{n}\left(g\left(\sigma_{a_{i}}^{-}\right)\right)^{\omega_{i}}\right)$

$\leq g^{-1}\left(\prod_{i=1}^{n}\left(g\left(\sigma_{a_{i}}^{+}\right)\right)^{\omega_{i}}\right) \leq g^{-1}\left(\prod_{i=1}^{n} g(9)^{\omega_{i}}\right)=9 \sum_{i=1}^{n} \omega_{i}=9$.

$$
\begin{aligned}
& h^{-1}\left(\prod_{i=1}^{n}\left(h\left(\rho_{a_{i}}^{+}\right)\right)^{\omega_{i}}\right) \cdot g^{-1}\left(\prod_{i=1}^{n}\left(g\left(\sigma_{a_{i}}^{+}\right)\right)^{\omega_{i}}\right) \\
& \quad \leq h^{-1}\left(\prod_{i=1}^{n}\left(h\left(\rho_{a_{i}}^{+}\right)\right)^{\omega_{i}}\right) \cdot g^{-1}\left(\prod_{i=1}^{n}\left(g\left(1 / \rho_{a_{i}}^{+}\right)\right)^{\omega_{i}}\right) \\
& \quad=h^{-1}\left(\sum_{i=1}^{n} \omega_{i} h\left(\rho_{a_{i}}^{+}\right)\right) \times\left(1 / h^{-1}\left(\sum_{i=1}^{n} \omega_{i} h\left(\rho_{a_{i}}^{+}\right)\right)\right)=1 .
\end{aligned}
$$

Based on the pseudo-multiplication, we can prove the following desirable properties of the IVIMWA operator.

Property 1 If all IVIMNs $\tilde{a}_{i}(i=1,2, \ldots, n)$ are equal, i.e., $\tilde{a}_{i}=\tilde{a}=\left(\tilde{\rho}_{a}, \tilde{\sigma}_{a}\right)$ for all $i$, then

$\operatorname{IVIMWA}\left(\tilde{a}_{1}, \tilde{a}_{2}, \ldots, \tilde{a}_{n}\right)=\tilde{a}$.

Proof Let $\tilde{a}_{i}=\tilde{a}=\left(\tilde{\rho}_{a}, \tilde{\sigma}_{a}\right)$, then

$\operatorname{IVIMWA}\left(\tilde{a}_{1}, \tilde{a}_{2}, \ldots, \tilde{a}_{n}\right)=\operatorname{IVIMWA}(\tilde{a}, \tilde{a}, \ldots, \tilde{a})=\sum_{i=1}^{n} \omega_{i} \tilde{a}$

$$
\begin{aligned}
& =\left(\left[h^{-1}\left(\prod_{i=1}^{n}\left(h\left(\rho_{a}^{-}\right)\right)^{\omega_{i}}\right), h^{-1}\left(\prod_{i=1}^{n}\left(h\left(\rho_{a}^{+}\right)\right)^{\omega_{i}}\right)\right],\right. \\
& \left.\times\left[g^{-1}\left(\prod_{i=1}^{n}\left(g\left(\sigma_{a}^{-}\right)\right)^{\omega_{i}}\right), g^{-1}\left(\prod_{i=1}^{n}\left(g\left(\sigma_{a}^{+}\right)\right)^{\omega_{i}}\right)\right]\right) \\
& =\left(\left[h^{-1}\left(h\left(\rho_{a}^{-}\right)\right), h^{-1}\left(h\left(\rho_{a}^{+}\right)\right)\right],\left[g^{-1}\left(g\left(\sigma_{a}^{-}\right)\right), g^{-1}\left(g\left(\sigma_{a}^{+}\right)\right)\right]\right) \\
& =\left(\left[\rho_{a}^{-}, \rho_{a}^{+}\right],\left[\sigma_{a}^{-}, \sigma_{a}^{+}\right]\right)=\tilde{a} .
\end{aligned}
$$


Property 1 can be called the idempotence law of the IVIMWA operator.

Property 2 Let $\tilde{a}_{i}=\left(\tilde{\rho}_{a_{i}}, \tilde{\sigma}_{a_{i}}\right)$ and $\tilde{b}_{i}=\left(\tilde{\rho}_{b_{i}}, \tilde{\sigma}_{b_{i}}\right) \quad(i=$ $1,2, \ldots, n)$ be two collections of IVIMNs, if $\rho_{a_{i}}^{-} \leq \rho_{b_{i}}^{-}$, $\rho_{a_{i}}^{+} \leq \rho_{b_{i}}^{+}, \sigma_{a_{i}}^{-} \geq \sigma_{b_{i}}^{-}$and $\sigma_{a_{i}}^{+} \geq \sigma_{b_{i}}^{+}$, for all $i$, then

$\operatorname{IVIMWA}\left(\tilde{a}_{1}, \tilde{a}_{2}, \ldots, \tilde{a}_{n}\right) \leq \operatorname{IVIMWA}\left(\tilde{b}_{1}, \tilde{b}_{2}, \ldots, \tilde{b}_{n}\right)$

Proof Due to $h(t)=g(1 / t)$, and $g(t):(0,+\infty) \rightarrow$ $(0,+\infty)$ is a strictly decreasing function, $h(t)$ is a strictly increasing function. Since $\rho_{a_{i}}^{-} \leq \rho_{b_{i}}^{-}, \rho_{a_{i}}^{+} \leq \rho_{b_{i}}^{+}, \sigma_{a_{i}}^{-} \geq \sigma_{b_{i}}^{-}$and $\sigma_{a_{i}}^{+} \geq \sigma_{b_{i}}^{+}$, then

$$
\begin{aligned}
& h^{-1}\left(\sum_{i=1}^{n} \omega_{i} h\left(\rho_{a_{i}}^{-}\right)\right) \leq h^{-1}\left(\sum_{i=1}^{n} \omega_{i} h\left(\rho_{b_{i}}^{-}\right)\right) \\
& h^{-1}\left(\sum_{i=1}^{n} \omega_{i} h\left(\rho_{a_{i}}^{+}\right)\right) \leq h^{-1}\left(\sum_{i=1}^{n} \omega_{i} h\left(\rho_{b_{i}}^{+}\right)\right) \\
& g^{-1}\left(\sum_{i=1}^{n} \omega_{i} g\left(\sigma_{a_{i}}^{-}\right)\right) \geq g^{-1}\left(\sum_{i=1}^{n} \omega_{i} g\left(\sigma_{b_{i}}^{-}\right)\right) \\
& g^{-1}\left(\sum_{i=1}^{n} \omega_{i} g\left(\sigma_{a_{i}}^{+}\right)\right) \geq g^{-1}\left(\sum_{i=1}^{n} \omega_{i} g\left(\sigma_{b_{i}}^{+}\right)\right)
\end{aligned}
$$

Thus, based on Definition 2.2, we have $s\left(\operatorname{IVIMWA}\left(\tilde{a}_{1}, \tilde{a}_{2}, \ldots, \tilde{a}_{n}\right)\right) \leq s\left(\operatorname{IVIMWA}\left(\tilde{b}_{1}, \tilde{b}_{2}, \ldots, \tilde{b}_{n}\right)\right)$.

Property 2 tells us that if $S\left(\tilde{a}_{i}\right)<S\left(\tilde{b}_{i}\right)$, then IVIMWA $\left(\tilde{a}_{1}, \tilde{a}_{2}, \ldots, \tilde{a}_{n}\right) \leq \operatorname{IVIMWA}\left(\tilde{b}_{1}, \tilde{b}_{2}, \ldots, \tilde{b}_{n}\right)$. Namely, the IVIMWA operator is a monotonically increasing function with respect to the input argument variable.

Property 3 Let $\tilde{a}_{i}=\left(\tilde{\rho}_{a_{i}}, \tilde{\sigma}_{a_{i}}\right)(i=1,2, \ldots, n)$ be a collection of IVIMNs, if $\tilde{b}=\left(\tilde{\rho}_{b}, \tilde{\sigma}_{b}\right)$ is an IVIMN, then,

$$
\begin{aligned}
& \operatorname{IVIMWA}\left(\tilde{a}_{1}+\tilde{b}, \tilde{a}_{2}+\tilde{b}, \ldots, \tilde{a}_{n}+\tilde{b}\right) \\
& \quad=\operatorname{IVIMWA}\left(\tilde{a}_{1}, \tilde{a}_{2}, \ldots, \tilde{a}_{n}\right)+\tilde{b}
\end{aligned}
$$

Proof Based on Definition 2.3, we have

$$
\begin{gathered}
\tilde{a}_{i}+\tilde{b}=\left(\left[h^{-1}\left(h\left(\rho_{a_{i}}^{-}\right) \cdot h\left(\rho_{b}^{-}\right)\right), h^{-1}\left(h\left(\rho_{a_{i}}^{+}\right) \cdot h\left(\rho_{b}^{+}\right)\right)\right],\right. \\
\left.\left[g^{-1}\left(g\left(\sigma_{a_{i}}^{-}\right) \cdot g\left(\sigma_{b}^{-}\right)\right), g^{-1}\left(g\left(\sigma_{a_{i}}^{+}\right) \cdot g\left(\sigma_{b}^{+}\right)\right)\right]\right)
\end{gathered}
$$

Therefore,

$\operatorname{IVIMWA}\left(\tilde{a}_{1}+\tilde{b}, \tilde{a}_{2}+\tilde{b}, \ldots, \tilde{a}_{n}+\tilde{b}\right)$

$$
\begin{aligned}
& =\left(\left[h^{-1}\left(\prod_{i=1}^{n}\left(h\left(h^{-1}\left(h\left(\rho_{a_{i}}^{-}\right) \cdot h\left(\rho_{b}^{-}\right)\right)\right)\right)^{\omega_{i}}\right), h^{-1}\left(\prod_{i=1}^{n}\left(h\left(h^{-1}\left(h\left(\rho_{a_{i}}^{+}\right) \cdot h\left(\rho_{b}^{+}\right)\right)\right)\right)^{\omega_{i}}\right)\right],\right. \\
& \left.\left[g^{-1}\left(\prod_{i=1}^{n}\left(g\left(g^{-1}\left(g\left(\sigma_{a_{i}}^{-}\right) \cdot g\left(\sigma_{b}^{-}\right)\right)\right)\right)^{\omega_{i}}\right), g^{-1}\left(\prod_{i=1}^{n}\left(g\left(g^{-1}\left(g\left(\sigma_{a_{i}}^{+}\right) \cdot g\left(\sigma_{b}^{+}\right)\right)\right)\right)^{\omega_{i}}\right)\right]\right) \\
& =\left(\left[h^{-1}\left(\prod_{i=1}^{n}\left(h\left(\rho_{a_{i}}^{-}\right) \cdot h\left(\rho_{b}^{-}\right)\right)^{\omega_{i}}\right), h^{-1}\left(\prod_{i=1}^{n}\left(h\left(\rho_{a_{i}}^{+}\right) \cdot h\left(\rho_{b}^{+}\right)\right)^{\omega_{i}}\right)\right],\left[g^{-1}\left(\prod_{i=1}^{n}\left(g\left(\sigma_{a_{i}}^{-}\right) \cdot g\left(\sigma_{b}^{-}\right)\right)^{\omega_{i}}\right), g^{-1}\left(\prod_{i=1}^{n}\left(g\left(\sigma_{a_{i}}^{+}\right) \cdot g\left(\sigma_{b}^{+}\right)\right)^{\omega_{i}}\right)\right]\right)
\end{aligned}
$$

$\operatorname{IVIMWA}\left(\tilde{a}_{1}, \tilde{a}_{2}, \ldots, \tilde{a}_{n}\right)+\tilde{b}$

$$
\begin{aligned}
& =\left(\left[h^{-1}\left(\prod_{i=1}^{n}\left(h\left(\rho_{a_{i}}^{-}\right)\right)^{\omega_{i}}\right), h^{-1}\left(\prod_{i=1}^{n}\left(h\left(\rho_{a_{i}}^{+}\right)\right)^{\omega_{i}}\right)\right],\left[g^{-1}\left(\prod_{i=1}^{n}\left(g\left(\sigma_{a_{i}}^{-}\right)\right)^{\omega_{i}}\right), g^{-1}\left(\prod_{i=1}^{n}\left(g\left(\sigma_{a_{i}}^{+}\right)\right)^{\omega_{i}}\right)\right]\right)+\left(\left[\rho_{b}^{-}, \rho_{b}^{+}\right],\left[\sigma_{b}^{-}, \sigma_{b}^{+}\right]\right) \\
& =\left(\left[h^{-1}\left(h\left(h^{-1}\left(\prod_{i=1}^{n}\left(h\left(\rho_{a_{i}}^{-}\right)\right)^{\omega_{i}}\right)\right) \cdot h\left(\rho_{b}^{-}\right)\right), h^{-1}\left(h\left(h^{-1}\left(\prod_{i=1}^{n}\left(h\left(\rho_{a_{i}}^{+}\right)\right)^{\omega_{i}}\right)\right) \cdot h\left(\rho_{b}^{+}\right)\right)\right],\right. \\
& \left.\left[g^{-1}\left(g\left(g^{-1}\left(\prod_{i=1}^{n}\left(g\left(\sigma_{a_{i}}^{-}\right)\right)^{\omega_{i}}\right)\right) \cdot g\left(\sigma_{b}^{-}\right)\right), g^{-1}\left(g\left(g^{-1}\left(\prod_{i=1}^{n}\left(g\left(\sigma_{a_{i}}^{+}\right)\right)^{\omega_{i}}\right)\right) \cdot g\left(\sigma_{b}^{+}\right)\right)\right]\right) \\
& =\left(\left[h^{-1}\left(\prod_{i=1}^{n}\left(h\left(\rho_{a_{i}}^{-}\right)\right)^{\omega_{i}} \cdot h\left(\rho_{b}^{-}\right)\right), h^{-1}\left(\prod_{i=1}^{n}\left(h\left(\rho_{a_{i}}^{+}\right)\right)^{\omega_{i}} \cdot h\left(\rho_{b}^{+}\right)\right)\right],\left[g^{-1}\left(\prod_{i=1}^{n}\left(g\left(\sigma_{a_{i}}^{-}\right)\right)^{\omega_{i}} \cdot g\left(\sigma_{b}^{-}\right)\right), g^{-1}\left(\prod_{i=1}^{n}\left(g\left(\sigma_{a_{i}}^{+}\right)\right)^{\omega_{i}} \cdot g\left(\sigma_{b}^{+}\right)\right)\right]\right) \\
& =\left(\left[h^{-1}\left(\prod_{i=1}^{n}\left(h\left(\rho_{a_{i}}^{-}\right) \cdot h\left(\rho_{b}^{-}\right)\right)^{\omega_{i}}\right), h^{-1}\left(\prod_{i=1}^{n}\left(h\left(\rho_{a_{i}}^{+}\right) \cdot h\left(\rho_{b}^{+}\right)\right)^{\omega_{i}}\right)\right],\left[g^{-1}\left(\prod_{i=1}^{n}\left(g\left(\sigma_{a_{i}}^{-}\right) \cdot g\left(\sigma_{b}^{-}\right)\right)^{\omega_{i}}\right), g^{-1}\left(\prod_{i=1}^{n}\left(g\left(\sigma_{a_{i}}^{+}\right) \cdot g\left(\sigma_{b}^{+}\right)\right)^{\omega_{i}}\right)\right]\right)
\end{aligned}
$$


Granul. Compute. (2017) 2:387-407

395

Then we can get IVIMWA $\left(\tilde{a}_{1}+\tilde{b}, \tilde{a}_{2}+\tilde{b}, \ldots, \tilde{a}_{n}+\tilde{b}\right)=$ $\operatorname{IVIMWA}\left(\tilde{a}_{1}, \tilde{a}_{2}, \ldots, \tilde{a}_{n}\right)+\tilde{b}$.

Property 4 Let $\tilde{a}_{i}=\left(\tilde{\rho}_{a_{i}}, \tilde{\sigma}_{a_{i}}\right)(i=1,2, \ldots, n)$ be a collection of IVIMNs, if $r>0$, then

$\operatorname{IVIMWA}\left(r \tilde{a}_{1}, r \tilde{a}_{2}, \ldots, r \tilde{a}_{n}\right)=r \operatorname{IVIMWA}\left(\tilde{a}_{1}, \tilde{a}_{2}, \ldots, \tilde{a}_{n}\right)$.

(13) Proof According to Definition 4, we have $r \tilde{a}=\left(\left[h^{-1}\left(\left(h\left(\rho_{a}^{-}\right)\right)^{r}\right), h^{-1}\left(\left(h\left(\rho_{a}^{+}\right)\right)^{r}\right)\right]\right.$,
$\left.\left[g^{-1}\left(\left(g\left(\sigma_{a}^{-}\right)\right)^{r}\right), g^{-1}\left(\left(g\left(\sigma_{a}^{+}\right)\right)^{r}\right)\right]\right)$.

Then,

$\operatorname{IVIMWA}\left(r \tilde{a}_{1}, r \tilde{a}_{2}, \ldots, r \tilde{a}_{n}\right)$

$$
\begin{aligned}
& =\left(\left[h^{-1}\left(\prod_{i=1}^{n}\left(h\left(h^{-1}\left(h\left(\rho_{a_{i}}^{-}\right)\right)^{r}\right)\right)^{\omega_{i}}\right)\right.\right. \\
& \left.h^{-1}\left(\prod_{i=1}^{n}\left(h\left(h^{-1}\left(h\left(\rho_{a_{i}}^{+}\right)\right)^{r}\right)\right)^{\omega_{i}}\right)\right] \\
& {\left[g^{-1}\left(\prod_{i=1}^{n}\left(g\left(g^{-1}\left(g\left(\sigma_{a_{i}}^{-}\right)\right)^{r}\right)\right)^{\omega_{i}}\right)\right.} \\
& \left.\left.g^{-1}\left(\prod_{i=1}^{n}\left(g\left(g^{-1}\left(g\left(\sigma_{a_{i}}^{+}\right)\right)^{r}\right)\right)^{\omega_{i}}\right)\right]\right) \\
& =\left(\left[h^{-1}\left(\prod_{i=1}^{n}\left(h\left(\rho_{a_{i}}^{-}\right)\right)^{r \omega_{i}}\right), h^{-1}\left(\prod_{i=1}^{n}\left(h\left(\rho_{a_{i}}^{+}\right)\right)^{r \omega_{i}}\right)\right]\right. \\
& \left.\left[g^{-1}\left(\prod_{i=1}^{n}\left(g\left(\sigma_{a_{i}}^{-}\right)\right)^{r \omega_{i}}\right), g^{-1}\left(\prod_{i=1}^{n}\left(g\left(\sigma_{a_{i}}^{+}\right)\right)^{r \omega_{i}}\right)\right]\right) .
\end{aligned}
$$

On the other hand,

$\operatorname{rIVIMWA}\left(\tilde{a}_{1}, \tilde{a}_{2}, \ldots, \tilde{a}_{n}\right)$

$$
\begin{aligned}
= & \left(\left[h^{-1}\left(\left(h\left(h^{-1}\left(\prod_{i=1}^{n}\left(h\left(\rho_{a_{i}}^{-}\right)\right)^{\omega_{i}}\right)\right)\right)\right)^{r}\right),\right. \\
& \left.h^{-1}\left(\left(h\left(h^{-1}\left(\prod_{i=1}^{n}\left(h\left(\rho_{a_{i}}^{+}\right)\right)^{\omega_{i}}\right)\right)\right)\right)\right] \\
\times & {\left[g^{-1}\left(\left(g\left(g^{-1}\left(\prod_{i=1}^{n}\left(g\left(\sigma_{a_{i}}^{-}\right)\right)^{\omega_{i}}\right)\right)\right)\right)^{r}\right), } \\
& \left.\left.g^{-1}\left(\left(g\left(g^{-1}\left(\prod_{i=1}^{n}\left(g\left(\sigma_{a_{i}}^{+}\right)\right)^{\omega_{i}}\right)\right)\right)\right)\right]\right) .
\end{aligned}
$$

Then we can obtain $\operatorname{IVIMWA}\left(r \tilde{a}_{1}, r \tilde{a}_{2}, \ldots, r \tilde{a}_{n}\right)=$ $r \operatorname{IVIMWA}\left(\tilde{a}_{1}, \tilde{a}_{2}, \ldots, \tilde{a}_{n}\right)$.
Properties 3 and 4 imply that the IVIMWA operator satisfies the associativity and distributivity laws. According to Properties 3 and 4, we can get Property 5 easily:

Property 5 Let $\tilde{a}_{i}=\left(\tilde{\rho}_{a_{i}}, \tilde{\sigma}_{a_{i}}\right)(i=1,2, \ldots, n)$ be a collection of IVIMNs, if $r>0$ and $\tilde{b}=\left(\tilde{\rho}_{b}, \tilde{\sigma}_{b}\right)$ is an IVIMN, then

$$
\begin{aligned}
& \operatorname{IVIMWA}\left(r \tilde{a}_{1}+\tilde{b}, r \tilde{a}_{2}+\tilde{b}, \ldots, r \tilde{a}_{n}+\tilde{b}\right) \\
& \quad=r \operatorname{IVIMWA}\left(\tilde{a}_{1}, \tilde{a}_{2}, \ldots, \tilde{a}_{n}\right)+\tilde{b} .
\end{aligned}
$$

Property 6 Let $\tilde{a}_{i}=\left(\tilde{\rho}_{a_{i}}, \tilde{\sigma}_{a_{i}}\right)$ and $\tilde{b}_{i}=\left(\tilde{\rho}_{b_{i}}, \tilde{\sigma}_{b_{i}}\right) \quad(i=$ $1,2, \ldots, n)$ be two collections of IVIMNs, then $\operatorname{IVIMWA}\left(\tilde{a}_{1}+\tilde{b}_{1}, \tilde{a}_{2}+\tilde{b}_{2}, \ldots, \tilde{a}_{n}+\tilde{b}_{n}\right)$

$$
=\operatorname{IVIMWA}\left(\tilde{a}_{1}, \tilde{a}_{2}, \ldots, \tilde{a}_{n}\right)+\operatorname{IVIMWA}\left(\tilde{b}_{1}, \tilde{b}_{2}, \ldots, \tilde{b}_{n}\right) .
$$

(15)

Proof Based on Definition 2.3, we have

$$
\begin{aligned}
& \tilde{a}_{i}+\tilde{b}_{i}=\left(\left[h^{-1}\left(h\left(\rho_{a_{i}}^{-}\right) h\left(\rho_{b_{i}}^{-}\right)\right), h^{-1}\left(h\left(\rho_{a_{i}}^{+}\right) h\left(\rho_{b_{i}}^{+}\right)\right)\right],\right. \\
& \left.\left[g^{-1}\left(g\left(\sigma_{a_{i}}^{-}\right) g\left(\sigma_{b_{i}}^{-}\right)\right), g^{-1}\left(g\left(\sigma_{a_{i}}^{+}\right) g\left(\sigma_{b_{i}}^{+}\right)\right)\right]\right) .
\end{aligned}
$$

Then

$\operatorname{IVIMWA}\left(\tilde{a}_{1}+\tilde{b}_{1}, \tilde{a}_{2}+\tilde{b}_{2}, \ldots, \tilde{a}_{n}+\tilde{b}_{n}\right)$

$$
=\left(\left[h^{-1}\left(\prod_{i=1}^{n}\left(h\left(h^{-1}\left(h\left(\rho_{a_{i}}^{-}\right) \cdot h\left(\rho_{b_{i}}^{-}\right)\right)\right)\right)^{\omega_{i}}\right)\right.\right. \text {, }
$$

$\left.h^{-1}\left(\prod_{i=1}^{n}\left(h\left(h^{-1}\left(h\left(\rho_{a_{i}}^{+}\right) \cdot h\left(\rho_{b_{i}}^{+}\right)\right)\right)\right)^{\omega_{i}}\right)\right]$,

$\left[g^{-1}\left(\prod_{i=1}^{n}\left(g\left(g^{-1}\left(g\left(\sigma_{a_{i}}^{-}\right) \cdot g\left(\sigma_{b_{i}}^{-}\right)\right)\right)\right)^{\omega_{i}}\right)\right.$,

$\left.\left.g^{-1}\left(\prod_{i=1}^{n}\left(g\left(g^{-1}\left(g\left(\sigma_{a_{i}}^{+}\right) \cdot g\left(\sigma_{b_{i}}^{+}\right)\right)\right)\right)^{\omega_{i}}\right)\right]\right)$

$=\left(\left[h^{-1}\left(\prod_{i=1}^{n}\left(h\left(\rho_{a_{i}}^{-}\right) \cdot h\left(\rho_{b_{i}}^{-}\right)\right)^{\omega_{i}}\right)\right.\right.$,

$\left.h^{-1}\left(\prod_{i=1}^{n}\left(h\left(\rho_{a_{i}}^{+}\right) \cdot h\left(\rho_{b_{i}}^{+}\right)\right)^{\omega_{i}}\right)\right]$,

$\left[g^{-1}\left(\prod_{i=1}^{n}\left(g\left(\sigma_{a_{i}}^{-}\right) \cdot g\left(\sigma_{b_{i}}^{-}\right)\right)^{\omega_{i}}\right)\right.$,

$\left.\left.g^{-1}\left(\prod_{i=1}^{n}\left(g\left(\sigma_{a_{i}}^{+}\right) \cdot g\left(\sigma_{b_{i}}^{+}\right)\right)^{\omega_{i}}\right)\right]\right)$

Springer 
$\operatorname{IVIMWA}\left(\tilde{a}_{1}, \tilde{a}_{2}, \ldots, \tilde{a}_{n}\right)+\operatorname{IVIMWA}\left(\tilde{b}_{1}, \tilde{b}_{2}, \ldots, \tilde{b}_{n}\right)$

$$
\begin{aligned}
& =\left(\left[h^{-1}\left(\prod_{i=1}^{n}\left(h\left(\rho_{a_{i}}^{-}\right)\right)^{\omega_{i}}\right), h^{-1}\left(\prod_{i=1}^{n}\left(h\left(\rho_{a_{i}}^{+}\right)\right)^{\omega_{i}}\right)\right],\left[g^{-1}\left(\prod_{i=1}^{n}\left(g\left(\sigma_{a_{i}}^{-}\right)\right)^{\omega_{i}}\right), g^{-1}\left(\prod_{i=1}^{n}\left(g\left(\sigma_{a_{i}}^{+}\right)\right)^{\omega_{i}}\right)\right]\right) \\
& +\left(\left[h^{-1}\left(\prod_{i=1}^{n}\left(h\left(\rho_{b_{i}}^{-}\right)\right)^{\omega_{i}}\right), h^{-1}\left(\prod_{i=1}^{n}\left(h\left(\rho_{b_{i}}^{+}\right)\right)^{\omega_{i}}\right)\right],\left[g^{-1}\left(\prod_{i=1}^{n}\left(g\left(\sigma_{b_{i}}^{-}\right)\right)^{\omega_{i}}\right), g^{-1}\left(\prod_{i=1}^{n}\left(g\left(\sigma_{b_{i}}^{+}\right)\right)^{\omega_{i}}\right)\right]\right) \\
& =\left(\left[h^{-1}\left(h\left(h^{-1}\left(\prod_{i=1}^{n}\left(h\left(\rho_{a_{i}}^{-}\right)\right)^{\omega_{i}}\right)\right) h\left(h^{-1}\left(\prod_{i=1}^{n}\left(h\left(\rho_{b_{i}}^{-}\right)\right)^{\omega_{i}}\right)\right)\right), h^{-1}\left(h\left(h^{-1}\left(\prod_{i=1}^{n}\left(h\left(\rho_{a_{i}}^{+}\right)\right)^{\omega_{i}}\right)\right) h\left(h^{-1}\left(\prod_{i=1}^{n}\left(h\left(\rho_{b_{i}}^{+}\right)\right)^{\omega_{i}}\right)\right)\right),\right.\right. \\
& \left.\left[g^{-1}\left(g\left(g^{-1}\left(\prod_{i=1}^{n}\left(g\left(\sigma_{a_{i}}^{-}\right)\right)^{\omega_{i}}\right)\right) g\left(g^{-1}\left(\prod_{i=1}^{n}\left(g\left(\sigma_{b_{i}}^{-}\right)\right)^{\omega_{i}}\right)\right)\right), g^{-1}\left(g\left(g^{-1}\left(\prod_{i=1}^{n}\left(g\left(\sigma_{a_{i}}^{+}\right)\right)^{\omega_{i}}\right)\right) g\left(g^{-1}\left(\prod_{i=1}^{n}\left(g\left(\sigma_{b_{i}}^{+}\right)\right)^{\omega_{i}}\right)\right)\right)\right]\right) \\
& =\left(\left[h^{-1}\left(\prod_{i=1}^{n}\left(h\left(\rho_{a_{i}}^{-}\right) \cdot h\left(\rho_{b_{i}}^{-}\right)\right)^{\omega_{i}}\right), h^{-1}\left(\prod_{i=1}^{n}\left(h\left(\rho_{a_{i}}^{+}\right) \cdot h\left(\rho_{b_{i}}^{+}\right)\right)^{\omega_{i}}\right)\right],\left[g^{-1}\left(\prod_{i=1}^{n}\left(g\left(\sigma_{a_{i}}^{-}\right) \cdot g\left(\sigma_{b_{i}}^{-}\right)\right)^{\omega_{i}}\right), g^{-1}\left(\prod_{i=1}^{n}\left(g\left(\sigma_{a_{i}}^{+}\right) \cdot g\left(\sigma_{b_{i}}^{+}\right)\right)\right)^{\omega_{i}}\right)\right] .
\end{aligned}
$$

Good properties of IVIMNs have laid the foundation for our further investigation.

If the function $g$ is assigned different forms, some specific IVIMWA operators can be obtained as follows:

Case 1 If $g(t)=(1+t) / t$, then $h(t)=t+1$, the IVIMWA operator reduces to

$\operatorname{IVIMWA}\left(\tilde{a}_{1}, \tilde{a}_{2}, \ldots, \tilde{a}_{n}\right)=\left(\left[\prod_{i=1}^{n}\left(1+\rho_{a_{i}}^{-}\right)^{\omega_{i}}-1, \prod_{i=1}^{n}\left(1+\rho_{a_{i}}^{+}\right)^{\omega_{i}}-1\right]\right.$,

$$
\left.\left[\frac{\prod_{i=1}^{n}\left(\sigma_{a_{i}}^{-}\right)^{\omega_{i}}}{\prod_{i=1}^{n}\left(1+\sigma_{a_{i}}^{-}\right)^{\omega_{i}}-\prod_{i=1}^{n}\left(\sigma_{a_{i}}^{-}\right)^{\omega_{i}}}, \frac{\prod_{i=1}^{n}\left(\sigma_{a_{i}}^{+}\right)^{\omega_{i}}}{\prod_{i=1}^{n}\left(1+\sigma_{a_{i}}^{+}\right)^{\omega_{i}}-\prod_{i=1}^{n}\left(\sigma_{a_{i}}^{+}\right)^{\omega_{i}}}\right]\right) .
$$

Case 1 is the same as the IMIFWA operator in ( $\mathrm{Yu}$ et al. 2013).

Case 2 If $g(t)=(2+t) / t$, then $h(t)=2 t+1$, the IVIMWA operator reduces to

$\operatorname{IVIMWA}\left(\tilde{a}_{1}, \tilde{a}_{2}, \ldots, \tilde{a}_{n}\right)$

$=\left(\left[\frac{\prod_{i=1}^{n}\left(1+2 \rho_{a_{i}}^{-}\right)^{\omega_{i}}-1}{2}, \frac{\prod_{i=1}^{n}\left(1+2 \rho_{a_{i}}^{+}\right)^{\omega_{i}}-1}{2}\right]\right.$,

$\left.\left[\frac{2 \prod_{i=1}^{n}\left(\sigma_{a_{i}}^{-}\right)^{\omega_{i}}}{\prod_{i=1}^{n}\left(2+\sigma_{a_{i}}^{-}\right)^{\omega_{i}}-\prod_{i=1}^{n}\left(\sigma_{a_{i}}^{-}\right)^{\omega_{i}}}, \frac{2 \prod_{i=1}^{n}\left(\sigma_{a_{i}}^{+}\right)^{\omega_{i}}}{\prod_{i=1}^{n}\left(2+\sigma_{a_{i}}^{+}\right)^{\omega_{i}}-\prod_{i=1}^{n}\left(\sigma_{a_{i}}^{+}\right)^{\omega_{i}}}\right]\right)$

Case 3 If $g(t)=(\gamma+t) / t, \gamma>0$, then $h(t)=\gamma t+1$, the IVIMWA operator reduces to

$$
\begin{aligned}
& \operatorname{IVIMWA}\left(\tilde{a}_{1}, \tilde{a}_{2}, \ldots, \tilde{a}_{n}\right) \\
& =\left(\left[\frac{\prod_{i=1}^{n}\left(1+\gamma \rho_{a_{i}}^{-}\right)^{\omega_{i}}-1}{\gamma}, \frac{\prod_{i=1}^{n}\left(1+\gamma \rho_{a_{i}}^{+}\right)^{\omega_{i}}-1}{\gamma}\right],\right. \\
& \left.\left[\frac{\gamma \prod_{i=1}^{n}\left(\sigma_{a_{i}}^{-}\right)^{\omega_{i}}}{\prod_{i=1}^{n}\left(\gamma+\sigma_{a_{i}}^{-}\right)^{\omega_{i}}-\prod_{i=1}^{n}\left(\sigma_{a_{i}}^{-}\right)^{\omega_{i}}}, \frac{\gamma \prod_{i=1}^{n}\left(\sigma_{a_{i}}^{+}\right)^{\omega_{i}}}{\prod_{i=1}^{n}\left(\gamma+\sigma_{a_{i}}^{+}\right)^{\omega_{i}}-\prod_{i=1}^{n}\left(\sigma_{a_{i}}^{+}\right)^{\omega_{i}}}\right]\right) .
\end{aligned}
$$

Especially, if $\gamma=1$, then Eq. (18) reduces to Eq. (16); if $\gamma=2$, then Eq. (18) reduces to Eq. (17).

Motivated by the ordered weighted averaging (OWA) operator (Yager 1988), we can define the following aggregation operator:

Definition 4.2 (Yu et al. 2013) Let $\tilde{a}_{i}=\left(\tilde{\rho}_{a_{i}}, \tilde{\sigma}_{a_{i}}\right)(i=$ $1,2, \ldots, n)$ be a collection of IVIMNs, and $\omega=$ $\left(\omega_{1}, \omega_{2}, \ldots, \omega_{n}\right)^{T}$ be the weight vector of $\tilde{a}_{\text {ind }(i)}$ such that $\omega_{i} \in[0,1]$ and $\sum_{i=1}^{n} \omega_{i}=1$, if

$\operatorname{IVIMOWA}\left(\tilde{a}_{1}, \tilde{a}_{2}, \ldots, \tilde{a}_{n}\right)=\sum_{i=1}^{n} \omega_{i} \tilde{a}_{\operatorname{ind}(i)}$

then the function IVIMOWA is called the interval-valued intuitionistic multiplicative ordered weighted averaging (IVIMOWA) operator, where $\operatorname{ind}(i): \quad\{i=1,2, \ldots, n\} \rightarrow$ $\{i=1,2, \ldots, n\}$ is a non-increasing permutation, such that $\tilde{a}_{\text {ind }(i)} \geq \tilde{a}_{\text {ind }(i+1)}$. Without ambiguousness, ind $(i)$ can be simplified as $(i)$, such that $\tilde{a}_{(i)}$ is the $i$-th largest $\tilde{a}_{i}$. Similarly, here we call the IMIFOWA operator in (Yu et al. 2013) as the IVIMWA operator with regard to the homogeneity of the terms.

Especially, if $\omega=(1 / n, 1 / n, \ldots, 1 / n)^{T}$, then the IVIMOWA operator reduces to the IVIMA operator of IVIMNs (Eq. (8)).

In a similar way, based on Definition 4.2, we have

Theorem 4.2 Let $\tilde{a}_{i}=\left(\tilde{\rho}_{a_{i}}, \tilde{\sigma}_{a_{i}}\right)(i=1,2, \ldots, n)$ be $a$ collection of IVIMNs, and $(i):\{i=1,2, \ldots, n\} \rightarrow$ $\{i=1,2, \ldots, n\}$ be a non-increasing permutation such that $\tilde{a}_{(i)} \geq \tilde{a}_{(i+1)}$. Suppose that $\omega=\left(\omega_{1}, \omega_{2}, \ldots, \omega_{n}\right)^{T}$ is the weight vector of $\tilde{a}_{(i)}$, satisfying $\omega_{i} \in[0,1]$ and $\sum_{i=1}^{n} \omega_{i}=$ 1 , then the aggregated value by using the IVIMOWA operator is also an IVIMN, and 


$$
\begin{aligned}
& \operatorname{IVIMOWA}\left(\tilde{a}_{1}, \tilde{a}_{2}, \ldots, \tilde{a}_{n}\right)=\sum_{i=1}^{n} \omega_{i} \tilde{a}_{(i)} \\
& =\left(\left[h^{-1}\left(\prod_{i=1}^{n}\left(h\left(\rho_{a_{(i)}}^{-}\right)\right)^{\omega_{i}}\right), h^{-1}\left(\prod_{i=1}^{n}\left(h\left(\rho_{a_{(i)}}^{+}\right)\right)^{\omega_{i}}\right)\right],\right. \\
& \left.\left[g^{-1}\left(\prod_{i=1}^{n}\left(g\left(\sigma_{a_{(i)}}^{-}\right)\right)^{\omega_{i}}\right), g^{-1}\left(\prod_{i=1}^{n}\left(g\left(\sigma_{a_{(i)}}^{+}\right)\right)^{\omega_{i}}\right)\right]\right) .
\end{aligned}
$$

The proof is similar to that of Theorem 4.1, here we omit it for the space's limit.

Furthermore, we can easily get that the IVIMOWA operator satisfies Properties 1-6, and it also has the following property:

Property 7 Let $\tilde{a}_{i}=\left(\tilde{\rho}_{a_{i}}, \tilde{\sigma}_{a_{i}}\right)(i=1,2, \ldots, n)$ be a collection of IVIMNs, if $\tilde{a}_{\xi(i)}(i=1,2, \ldots, n)$ is any permutation of $\tilde{a}_{i}$, then

$\operatorname{IVIMOWA}\left(\tilde{a}_{1}, \tilde{a}_{2}, \ldots, \tilde{a}_{n}\right)$

$=\operatorname{IVIMOWA}\left(\tilde{a}_{\xi(1)}, \tilde{a}_{\xi(2)}, \ldots, \tilde{a}_{\xi(n)}\right)$.

If the function $\mathrm{g}$ is assigned different forms, then some specific IVIMOWA operators can be obtained as follows, where $\tilde{a}_{(i)}$ is a non-increasing permutation of $\mathrm{a}_{\mathrm{i}}(\mathrm{i}=1,2, \ldots, \mathrm{n})$.

Case $\mathbf{1}^{\prime}$ If $g(t)=(1+t) / t$, then $h(t)=t+1$, the IVIMOWA operator reduces to

$\operatorname{IVIMOWA}\left(\tilde{a}_{1}, \tilde{a}_{2}, \ldots, \tilde{a}_{n}\right)$

$$
\begin{aligned}
& =\left(\left[\prod_{i=1}^{n}\left(1+\rho_{a_{(i)}}^{-}\right)^{\omega_{i}}-1, \prod_{i=1}^{n}\left(1+\rho_{a_{(i)}}^{+}\right)^{\omega_{i}}-1\right],\right. \\
& \left.\left[\frac{\prod_{i=1}^{n}\left(\sigma_{a_{(i)}}^{-}\right)^{\omega_{i}}}{\prod_{i=1}^{n}\left(1+\sigma_{a_{(i)}}^{-}\right)^{\omega_{i}}-\prod_{i=1}^{n}\left(\sigma_{a_{(i)}}^{-}\right)^{\omega_{i}}}, \frac{\prod_{i=1}^{n}\left(\sigma_{a_{(i)}}^{+}\right)^{\omega_{i}}}{\prod_{i=1}^{n}\left(1+\sigma_{a_{(i)}}^{+}\right)^{\omega_{i}}-\prod_{i=1}^{n}\left(\sigma_{a_{(i)}}^{+}\right)^{\omega_{i}}}\right]\right) .
\end{aligned}
$$

Cased $1^{\prime}$ is the same as the IMIFOWA operator in $(\mathrm{Yu}$ et al. 2013).

Case $2^{\prime}$ If $g(t)=(2+t) / t$, then $h(t)=2 t+1$, the IVIMOWA operator reduces to

$$
\begin{aligned}
& \operatorname{IVIMOWA}\left(\tilde{a}_{1}, \tilde{a}_{2}, \ldots, \tilde{a}_{n}\right) \\
& =\left(\left[\frac{\prod_{i=1}^{n}\left(1+2 \rho_{a_{(i)}}^{-}\right)^{\omega_{i}}-1}{2}, \frac{\prod_{i=1}^{n}\left(1+2 \rho_{a_{(i)}}^{+}\right)^{\omega_{i}}-1}{2}\right],\right. \\
& \left.\left[\frac{2 \prod_{i=1}^{n}\left(\sigma_{a_{(i)}}^{-}\right)^{\omega_{i}}}{\prod_{i=1}^{n}\left(2+\sigma_{a_{(i)}}^{-}\right)^{\omega_{i}}-\prod_{i=1}^{n}\left(\sigma_{a_{(i)}}^{-}\right)^{\omega_{i}}}, \frac{2 \prod_{i=1}^{n}\left(\sigma_{a_{(i)}}^{+}\right)^{\omega_{i}}}{\prod_{i=1}^{n}\left(2+\sigma_{a_{(i)}}^{+}\right)^{\omega_{i}}-\prod_{i=1}^{n}\left(\sigma_{a_{(i)}}^{+}\right)^{\omega_{i}}}\right]\right) .
\end{aligned}
$$

Case $3^{\prime}$ If $g(t)=(\gamma+t) / t, \gamma>0$, then $h(t)=\gamma t+1$, the IVIMOWA operator reduces to

$$
\begin{aligned}
& \operatorname{IVIMOWA}\left(\tilde{a}_{1}, \tilde{a}_{2}, \ldots, \tilde{a}_{n}\right) \\
& =\left(\left[\frac{\prod_{i=1}^{n}\left(1+\gamma \rho_{a_{(i)}}^{-}\right)^{\omega_{i}}-1}{\gamma}, \frac{\prod_{i=1}^{n}\left(1+\gamma \rho_{a_{(i)}}^{+}\right)^{\omega_{i}}-1}{\gamma}\right],\right. \\
& \left.\left[\frac{\gamma \prod_{i=1}^{n}\left(\sigma_{a_{(i)}}^{-}\right)^{\omega_{i}}}{\prod_{i=1}^{n}\left(\gamma+\sigma_{a_{(i)}}^{-}\right)^{\omega_{i}}-\prod_{i=1}^{n}\left(\sigma_{a_{(i)}}^{-}\right)^{\omega_{i}}}, \frac{\gamma \prod_{i=1}^{n}\left(\sigma_{a_{(i)}}^{+}\right)^{\omega_{i}}}{\prod_{i=1}^{n}\left(\gamma+\sigma_{a_{(i)}}^{+}\right)^{\omega_{i}}-\prod_{i=1}^{n}\left(\sigma_{a_{(i)}}^{+}\right)^{\omega_{i}}}\right]\right) .
\end{aligned}
$$

Especially, if $\gamma=1$, then Eq. (24) reduces to Eq. (22); if $\gamma=2$, then Eq. (24) reduces to Eq. (23).

The characteristic of the IVIMOWA operator is that it first reorders the given IVIMNs in descending order, and then aggregates all the ordered IVIMNs. In other words, a particular input $\tilde{a}_{i}$ is not associated with a particular weight $\omega_{i}$, whereas a particular ordered position $(i)$ is associated with a particular weight $\omega_{i}$. Thus, the IVIMWA operator emphasizes the IVIMNs themselves, while the IVIMOWA operator weights the ordered position of the IVIMNs.

We can easily find that the IMIFWA and IMIFOWA operators in (Yu et al. 2013) are two specific examples $(\gamma=1$ which is the simplest case) of the IVIMWA and IVIMOWA operators in this paper.

Next, we give an example to illustrate the proposed aggregation operators:

Example 2 Let $\tilde{a}_{i}=\left(\tilde{\rho}_{a_{i}}, \tilde{\sigma}_{a_{i}}\right)(i=1,2,3)$ be a collection of IVIMNs such that

$\tilde{a}_{1}=([3,5],[1 / 6,1 / 5])$,

$\tilde{a}_{2}=([1 / 6,1 / 3],[1 / 8,1 / 7])$,

$\tilde{a}_{3}=([2,3],[1 / 8,1 / 6])$,

and $\omega=(1 / 4,1 / 2,1 / 4)^{T}$ be the weight vector of $\tilde{a}_{i}$. If we utilize the IVIMWA operator to aggregate the three IVIMNs:

Case 1 Let $\gamma=1$, then based on Eq. (16), we have

$$
\begin{aligned}
\tilde{a}_{\gamma=1} & =\operatorname{IVIMWA}\left(\tilde{a}_{1}, \tilde{a}_{2}, \tilde{a}_{3}\right) \\
& =([1.0103,1.5558],[0.1342,0.1613]) .
\end{aligned}
$$

Case 2 Let $\gamma=2$, then based on Eq. (17), we have

$$
\begin{aligned}
\tilde{a}_{\gamma=2} & =\operatorname{IVIMWA}\left(\tilde{a}_{1}, \tilde{a}_{2}, \tilde{a}_{3}\right) \\
& =([0.9043,1.4121],[0.1343,0.1614]) .
\end{aligned}
$$

Case 3 Let $\gamma=10$, then based on Eq. (18), we have

$$
\begin{aligned}
\tilde{a}_{\gamma=10} & =\operatorname{IVIMWA}\left(\tilde{a}_{1}, \tilde{a}_{2}, \tilde{a}_{3}\right) \\
& =([0.7249,1.2126],[0.1343,0.1615]) .
\end{aligned}
$$

In order to observe the variations of the IVIMWA operator with respect to the parameter $\gamma$, we assign $\gamma$ different values $0<\gamma \leq 50$. Then the variations of the values 


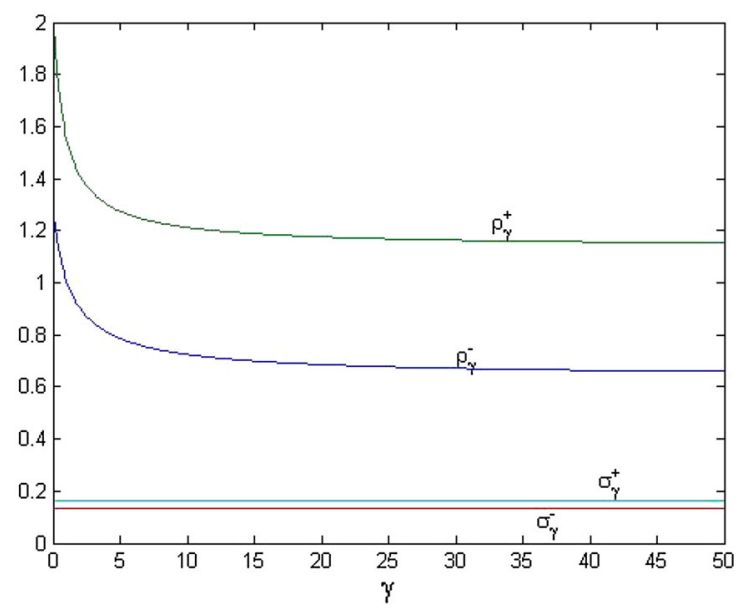

Fig. $1 \rho_{\gamma}^{-}, \rho_{\gamma}^{+}, \sigma_{\gamma}^{-}$, and $\sigma_{\gamma}^{+}$of $\tilde{a}_{0<\gamma \leq 50}$ with the IVIMWA operator

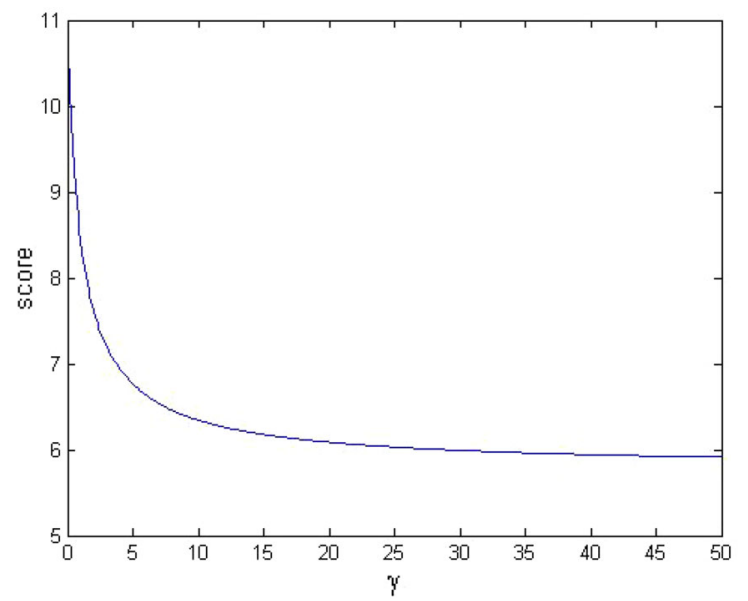

Fig. 2 The scores of $\tilde{a}_{0<\gamma \leq 50}$ with the IVIMWA operator

$\rho_{\gamma}^{-}, \rho_{\gamma}^{+}, \sigma_{\gamma}^{-}$, and $\sigma_{\gamma}^{+}$can be found clearly in Fig. 1, respectively. For clear observation, the scores of $\tilde{a}_{0<\gamma \leq 50}$ are presented in Fig. 2. If we utilize the IVIMOWA operator to aggregate these IVIMNs, then we need to rank the three IVIMNs according to the scores of $\tilde{a}_{i}$ which can be calculated:

$s\left(\tilde{a}_{1}\right)=21.2132, \quad s\left(\tilde{a}_{2}\right)=1.7638, \quad s\left(\tilde{a}_{3}\right)=16.9706$

by which we can obtain the new non-decreasing ranking:

$\tilde{a}_{(1)}=\tilde{a}_{1}, \quad \tilde{a}_{(2)}=\tilde{a}_{3}, \quad \tilde{a}_{(3)}=\tilde{a}_{2}$

Case $1^{\prime}$ Let $\gamma=1$, then based on Eq. (22), we have

$$
\begin{aligned}
\tilde{a}_{\gamma=1} & =\operatorname{IVIMOWA}\left(\tilde{a}_{1}, \tilde{a}_{2}, \tilde{a}_{3}\right) \\
& =([1.5457,2.3636],[0.1342,0.1677]) .
\end{aligned}
$$

Case $2^{\prime}$ Let $\gamma=2$, then based on Eq. (23), we have

$$
\begin{aligned}
\tilde{a}_{\gamma=2} & =\operatorname{IVIMOWA}\left(\tilde{a}_{1}, \tilde{a}_{2}, \tilde{a}_{3}\right) \\
& =([1.4542,2.2373],[0.1343,0.1678]) .
\end{aligned}
$$

Case $3^{\prime}$ Let $\gamma=10$, then based on Eq. (24), we have

$$
\begin{aligned}
\tilde{a}_{\gamma=10} & =\operatorname{IVIMOWA}\left(\tilde{a}_{1}, \tilde{a}_{2}, \tilde{a}_{3}\right) \\
& =([1.2818,2.0467],[0.1343,0.1678]) .
\end{aligned}
$$

Similarly, we assign the parameter $\gamma$ different values $0<\gamma \leq 50$ in order to observe the variations of the IVIMOWA operator with respect to the parameter $\gamma$. Then the variations of the values $\rho_{\gamma}^{-}, \rho_{\gamma}^{+}, \sigma_{\gamma}^{-}$, and $\sigma_{\gamma}^{+}$can be observed clearly in Fig. 3, respectively, and the scores of $\tilde{a}_{0<\gamma<50}$ are presented in Fig. 4 for clear observation.

Comparing Fig. 2 with Fig. 4, we can find that both the scores in Figs. 2 and 3 decrease as the value of the parameter $\gamma$ increases, and the scores obtained by utilizing the IVIMOWA operator (see Fig. 4) are larger than the ones obtained by using the IVIMWA operator (see Fig. 2). Obviously, the scores in Fig. 2 concentrate between 6 and 10, whereas all the scores in Fig. 4 are larger than 10, and concentrate between 10 and 14. Actually, according to the characteristic of the IVIMOWA operator, those largest and smallest IVIMNs ( $\tilde{a}_{1}$ and $\left.\tilde{a}_{2}\right)$ are assigned the smallest weights $\left(\omega_{1}=\omega_{3}=1 / 4\right)$, while the other IVIMN $\left(\tilde{a}_{3}\right)$ is assigned the largest weight $\left(\omega_{2}=1 / 2\right)$. Thus, the scores derived by the IVIMOWA operator are closer to the value of $s\left(\tilde{a}_{3}\right)\left(s\left(\tilde{a}_{3}\right)=16.9706\right)$ than the ones calculated by the IVIMWA operator.

\subsection{Some aggregation operators for the correlated inputs}

In this section, we mainly propose some aggregation operators to reflect the correlations or connections of the aggregated input IVIMNs based on Choquet integral (Beliakov et al. 2007; Choquet 1953; Grabisch 1996; Yager 2004) and Sugeno measure (also called $\lambda$-fuzzy measure) (Beliakov et al. 2007; Sugeno 1974). Choquet integral is a useful class of aggregation functions to model interactions between inputs. Choquet Integration was defined with respect to not necessarily additive monotone measure, called fuzzy measures. Fuzzy measure is an offspring of classical measure, and the Sugeno measure is one typical non-additive of them which is suitable for comprehensive evaluation problems which exist interactions. Different types of fuzzy measures would lead to specific features. The following Choquet integral-based aggregation operator is the combination of Choquet integral and Sugeno measure.

Firstly, we introduce some basic concepts:

Let $N=\{1,2, \ldots, n\}, \quad X=\left\{x_{1}, x_{2}, \ldots, x_{n}\right\}$ and $E=$ $\left\{e_{1}, e_{2}, \ldots, e_{n}\right\}$ be three finite sets. 


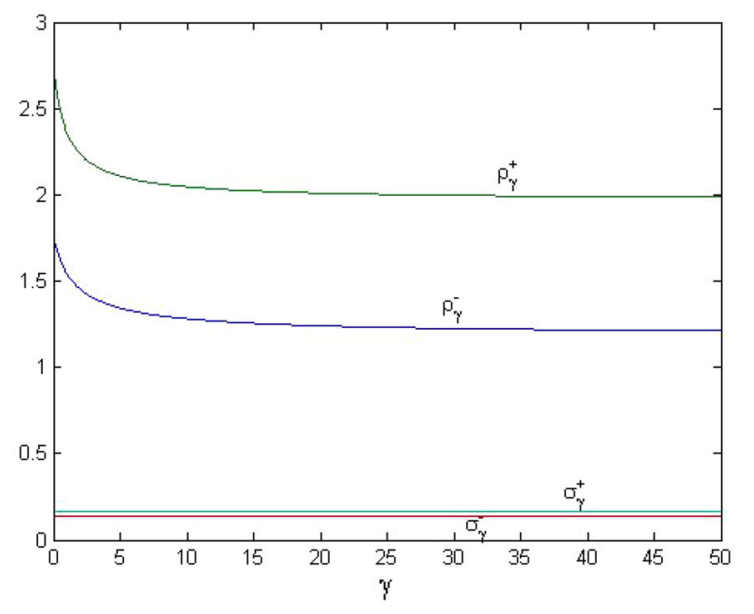

Fig. $3 \rho_{\gamma}^{-}, \rho_{\gamma}^{+}, \sigma_{\gamma}^{-}$, and $\sigma_{\gamma}^{+}$of $\tilde{a}_{0<\gamma \leq 50}$ with the IVIMOWA operator

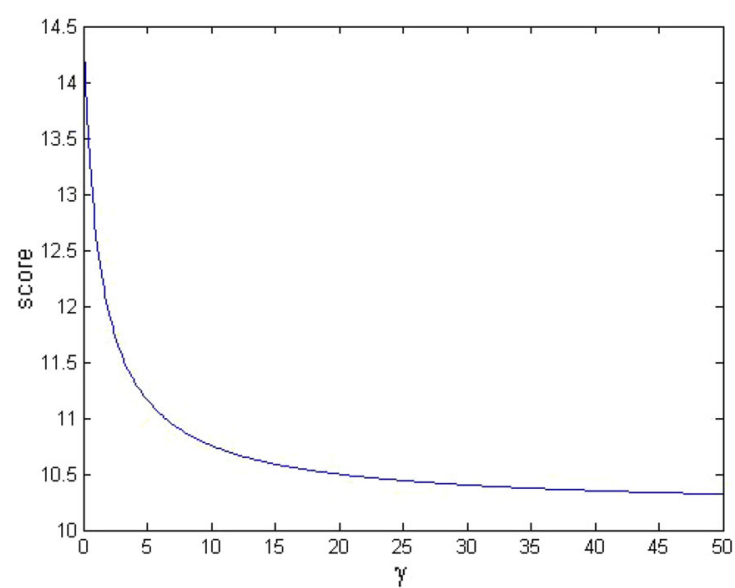

Fig. 4 The scores of $\tilde{a}_{0<\gamma \leq 50}$ with the IVIMOWA operator

Definition 4.3 (Sugeno 1974) A discrete fuzzy measure is a set function $\mu: 2^{N} \rightarrow[0,1]$ which is monotonic and satisfies $\mu(\emptyset)=0$ and $\mu(N)=1$.

Definition 4.4 (Beliakov et al. 2007; Grabisch 1996) The discrete Choquet integral with respect to a fuzzy measure $\mu$ is given by

$C_{\mu}(X)=\sum_{i=1}^{n} x_{(i)}\left[\mu\left(\left\{j \mid x_{j} \geq x_{(i)}\right\}\right)-\mu\left(\left\{j \mid x_{j} \geq x_{(i-1)}\right\}\right)\right]$,

where $\left\{x_{(1)}, x_{(2)}, \ldots, x_{(n)}\right\}$ is a non-increasing permutation of $X$, and $x_{(0)}=\infty$ by convention.

Definition 4.5 (Beliakov et al. 2007; Sugeno 1974) Given a parameter $\tau \in(-1, \infty)$, a Sugeno measure is a fuzzy measure $m$ on the set $E$, for all $P, Q \subseteq E, P \cap Q=\emptyset$, satisfying

$m(P \cup Q)=m(P)+m(Q)+\tau m(P) m(Q)$.
Suppose that $E_{j}\left(j=1,2, \ldots, 2^{n}\right)$ is any subset of $E$, then all values $m\left(E_{j}\right)$ can be easily computed from $n$ independent values $m\left(e_{i}\right)(i=1,2, \ldots, n)$, by utilizing the following explicit formula given by Sugeno (Sugeno 1974):

$m\left(E_{j}\right)= \begin{cases}\frac{1}{\tau}\left(\prod_{e_{i} \in E_{j}}\left(1+\tau m\left(e_{i}\right)\right)-1\right), & \tau \neq 0 \\ \sum_{e_{i} \in E_{j}} m\left(e_{i}\right), & \tau=0\end{cases}$

The value of $\tau$ can be uniquely determined from $m(E)=1$, which can be written as

$\tau+1=\prod_{i=1}^{n}\left(1+\tau m\left(e_{i}\right)\right)$

If we apply the Sugeno measure $m$ to the Choquet integral when all input arguments are IVIMNs, then we can define the Choquet integral-based aggregation operator for IVIMNs:

Definition 4.6 Let $\tilde{a}_{i}=\left(\tilde{\rho}_{a_{i}}, \tilde{\sigma}_{a_{i}}\right)(i=1,2, \ldots, n)$ be a collection of IVIMNs defined on the set $E=$ $\left\{e_{1}, e_{2}, \ldots, e_{n}\right\}$, if

$\mathrm{C}_{m^{-}} \operatorname{IVIM}\left(\tilde{a}_{1}, \tilde{a}_{2}, \ldots, \tilde{a}_{n}\right)=\sum_{i=1}^{n}\left(m\left(E_{i}\right)-m\left(E_{i-1}\right)\right) \cdot \tilde{a}_{(i)}$,

then the function $\mathrm{C}_{m}$-IVIM is called as the Choquet integral-based aggregation operator of the IVIMNs $\left(\mathrm{C}_{m}\right.$-IVIM operator), where $(i)$ is the index of $i$-th largest $\tilde{a}_{i}$, implying $\tilde{a}_{(i)} \geq \tilde{a}_{(i+1)}, E_{i}=\left\{e_{(1)}, \ldots, e_{(i)}\right\}$ and $m\left(E_{i}\right)$ is the Sugeno measure of $E_{i}$. We let $E_{0}=\emptyset$ by convention.

The $\mathrm{C}_{m}$-IVIM operator has the following properties:

Property 8 Let $\tilde{a}_{i}=\left(\tilde{\rho}_{a_{i}}, \tilde{\sigma}_{a_{i}}\right)$ and $\tilde{a}_{i}^{\prime}=\left(\tilde{\rho}_{a_{i}^{\prime}}, \tilde{\sigma}_{a_{i}^{\prime}}\right)(i=$ $1,2, \ldots, n)$ be two collections of IVIMNs, if $\tilde{a}_{i} \leq \tilde{a}_{i}^{\prime}$, then $\mathrm{C}_{m}-\operatorname{IVIM}\left(\tilde{a}_{1}, \tilde{a}_{2}, \ldots, \tilde{a}_{n}\right) \leq \mathrm{C}_{m^{-}} \operatorname{IVIM}\left(\tilde{a}_{1}^{\prime}, \tilde{a}_{2}^{\prime}, \ldots, \tilde{a}_{n}^{\prime}\right)$

namely, the $\mathrm{C}_{m}$-IVIM operator is a monotonically increasing function with respect to input argument variable.

Proof This proposition can be obtained by taking partial derivative of the $\mathrm{C}_{m}$-IVIM operator:

$$
\begin{aligned}
& \frac{\partial \mathrm{C}_{\mathrm{m}}-\operatorname{IVIM}\left(\tilde{a}_{1}, \tilde{a}_{2}, \ldots, \tilde{a}_{n}\right)}{\partial \tilde{a}_{i}} \\
& =\frac{\partial \sum_{i=1}^{n}\left(m\left(E_{i}\right)-m\left(E_{i-1}\right)\right) \cdot \tilde{a}_{(i)}}{\partial \tilde{a}_{(i)}} \\
& =m\left(E_{i}\right)-m\left(E_{i-1}\right) .
\end{aligned}
$$

Since $m(E)=1, m(\emptyset)=0$, and $E_{i} \subseteq E(i=1,2, \ldots, n)$, then $0<m\left(E_{i}\right) \leq 1$ and $0<m\left(e_{i}\right) \leq 1$. Therefore, $m\left(E_{i}\right)-$ $m\left(E_{i-1}\right)>0$, then we have 
$\frac{\partial \mathrm{C}_{\mathrm{m}}-\operatorname{IVIM}\left(\tilde{a}_{1}, \tilde{a}_{2}, \ldots, \tilde{a}_{n}\right)}{\partial \tilde{a}_{i}}>0$.

Furthermore, the following properties can be easily obtained:

Property 9 If all IVIMNs $\tilde{a}_{i}(i=1,2, \ldots, n)$ are equal, i.e., $\tilde{a}_{i}=\tilde{a}=\left(\tilde{\rho}_{a}, \tilde{\sigma}_{a}\right)$ for all $i$, then

$\mathrm{C}_{m}-\operatorname{IVIM}\left(\tilde{a}_{1}, \tilde{a}_{2}, \ldots, \tilde{a}_{n}\right)=\tilde{a}$.

Property 9 can be called the idempotence law of the $\mathrm{C}_{\mathrm{m}}{ }^{-}$ IVIM operator..

Property 10 Let $\tilde{a}_{i}=\left(\tilde{\rho}_{a_{i}}, \tilde{\sigma}_{a_{i}}\right)(i=1,2, \ldots, n)$ be $a$ collection of IVIMN, if $\tilde{a}_{\xi(i)}$ is any permutation of $\tilde{a}_{i}$, then $\mathrm{C}_{m^{-}} \operatorname{IVIM}\left(\tilde{a}_{1}, \tilde{a}_{2}, \ldots, \tilde{a}_{n}\right)=\mathrm{C}_{m^{-}} \operatorname{IVIM}\left(\tilde{a}_{\xi(1)}, \tilde{a}_{\xi(2)}, \ldots, \tilde{a}_{\xi(n)}\right)$.

Based on the IVIMOWA operator (Eq. (20)), we can have the following theorem:

Theorem 4.3 Let $\tilde{a}_{i}=\left(\tilde{\rho}_{a_{i}}, \tilde{\sigma}_{a_{i}}\right)(i=1,2, \ldots, n)$ be $a$ collection of IVIMNs defined on the set $E=$ $\left\{e_{1}, e_{2}, \ldots, e_{n}\right\}$, then the aggregated value by using the $\mathrm{C}_{m^{-}}$ IVIM operator is also an IVIMN, and

$$
\begin{aligned}
\mathrm{C}_{m}- & \operatorname{IVIM}\left(\tilde{a}_{1}, \tilde{a}_{2}, \ldots, \tilde{a}_{n}\right) \\
& =\left(\left[h^{-1}\left(\prod_{i=1}^{n}\left(h\left(\rho_{a_{(i)}}^{-}\right)\right)^{m\left(E_{i}\right)-m\left(E_{i-1}\right)}\right),\right.\right. \\
& \left.h^{-1}\left(\prod_{i=1}^{n}\left(h\left(\rho_{a_{(i)}}^{+}\right)\right)^{m\left(E_{i}\right)-m\left(E_{i-1}\right)}\right)\right], \\
& \times\left[g^{-1}\left(\prod_{i=1}^{n}\left(g\left(\sigma_{a_{(i)}}^{-}\right)\right)^{m\left(E_{i}\right)-m\left(E_{i-1}\right)}\right),\right. \\
& \left.\left.g^{-1}\left(\prod_{i=1}^{n}\left(g\left(\sigma_{a_{(i)}}^{+}\right)\right)^{m\left(E_{i}\right)-m\left(E_{i-1}\right)}\right)\right]\right),
\end{aligned}
$$

where $(i)$ is the index of $i$-th largest $\tilde{a}_{i}, E_{i}=$ $\left\{e_{(1)}, \ldots, e_{(i)}\right\}$, and $m\left(E_{i}\right)$ is the Sugeno measure of $E_{i}$. Let $E_{0}=\emptyset$ by convention .

Proof Based on Property 8, we have $m\left(E_{i}\right)-$ $m\left(E_{i-1}\right)>0$. Since $m(E)=1$ and $m(\emptyset)=0$, then $0<m\left(E_{i}\right)-m\left(E_{i-1}\right) \leq 1$ and $\sum_{i=1}^{n}\left(m\left(E_{i}\right)-m\left(E_{i-1}\right)\right)=$ $m(E)=1$, satisfying the conditions of the weight vector $\omega$ in Theorem 4.2 (Eq. (20)). The remaining steps of the proof are similar as that of Theorem 4.1; here, we omit it for the space's limit.
Actually, if we extend the Choquet integral to the IVIMOWA operator proposed in Sect. 4.1 by $\omega_{i}=m\left(E_{i}\right)-$ $m\left(E_{i-1}\right)$, we can obtain Eq. (32) as well.

Similar to Cases 1-3 and Cases $1^{\prime}-3^{\prime}$, we assign the function $g$ in Eq. (32) different forms, then some specific $\mathrm{C}_{m}$-IVIM operators can be got, where $\tilde{a}_{(i)}$ is the $i$-th largest $\tilde{a}_{i}, E_{i}=\left\{e_{(1)}, \ldots, e_{(i)}\right\}$, and $m\left(E_{i}\right)$ is the Sugeno measure of $E_{i}(i=1,2, \ldots, n)$ :

Case $1^{\prime \prime}$ If $g(t)=(1+t) / t$, then $h(t)=t+1$, the $\mathrm{C}_{m^{-}}$ IVIM operator reduces to

$$
\begin{aligned}
& \mathrm{C}_{m^{-}} \operatorname{IVIM}\left(\tilde{a}_{1}, \tilde{a}_{2}, \ldots, \tilde{a}_{n}\right)= \\
& \left(\left[\frac{\prod_{i=1}^{n}\left(1+\gamma \rho_{a_{(i)}}^{-}\right)^{m\left(E_{i}\right)-m\left(E_{i-1}\right)}-1}{\gamma}, \frac{\prod_{i=1}^{n}\left(1+\gamma \rho_{a_{(i)}}^{+}\right)^{m\left(E_{i}\right)-m\left(E_{i-1}\right)}-1}{\gamma}\right],\right. \\
& {\left[\frac{\gamma \prod_{i=1}^{n}\left(\sigma_{a_{(i)}}^{-}\right)^{m\left(E_{i}\right)-m\left(E_{i-1}\right)}}{\prod_{i=1}^{n}\left(\gamma+\sigma_{a_{(i)}}^{-}\right)^{m\left(E_{i}\right)-m\left(E_{i-1}\right)}-\prod_{i=1}^{n}\left(\sigma_{a_{(i)}}^{-}\right)^{m\left(E_{i}\right)-m\left(E_{i-1}\right)}},\right.} \\
& \left.\left.\frac{\gamma \prod_{i=1}^{n}\left(\sigma_{a_{(i)}}^{+}\right)^{m\left(E_{i)}\right)-m\left(E_{i-1}\right)}}{\prod_{i=1}^{n}\left(\gamma+\sigma_{a_{(i)}}^{+}\right)^{m\left(E_{i}\right)-m\left(E_{i-1}\right)}-\prod_{i=1}^{n}\left(\sigma_{a_{(i)}}^{+}\right)^{m\left(E_{i}\right)-m\left(E_{i-1}\right)}}\right]\right) .
\end{aligned}
$$

Case 2" If $g(t)=(2+t) / t$, then $h(t)=2 t+1$, and the $\mathrm{C}_{m}$-IVIM operator reduces to

$\mathrm{C}_{m}-\operatorname{IVIM}\left(\tilde{a}_{1}, \tilde{a}_{2}, \ldots, \tilde{a}_{n}\right)$

$$
\begin{aligned}
& =\left(\left[\frac{\prod_{i=1}^{n}\left(1+2 \rho_{a_{(i)}}^{-}\right)^{m\left(E_{i}\right)-m\left(E_{i-1}\right)}-1}{2}, \frac{\prod_{i=1}^{n}\left(1+2 \rho_{a_{(i)}}^{+}\right)^{m\left(E_{i}\right)-m\left(E_{i-1}\right)}-1}{2}\right],\right. \\
& {\left[\frac{2 \prod_{i=1}^{n}\left(\sigma_{a_{(i)}}^{-}\right)^{m\left(E_{i}\right)-m\left(E_{i-1}\right)}}{\prod_{i=1}^{n}\left(2+\sigma_{a_{(i)}}^{-}\right)^{m\left(E_{i}\right)-m\left(E_{i-1}\right)}-\prod_{i=1}^{n}\left(\sigma_{a_{(i)}}^{-}\right)^{m\left(E_{i}\right)-m\left(E_{i-1}\right)}},\right.} \\
& \left.\left.\frac{2 \prod_{i=1}^{n}\left(\sigma_{a_{(i)}}^{+}\right)^{m\left(E_{i}\right)-m\left(E_{i-1}\right)}}{\prod_{i=1}^{n}\left(2+\sigma_{a_{(i)}}^{+}\right)^{m\left(E_{i}\right)-m\left(E_{i-1}\right)}-\prod_{i=1}^{n}\left(\sigma_{a_{(i)}}^{+}\right)^{m\left(E_{i}\right)-m\left(E_{i-1}\right)}}\right]\right) .
\end{aligned}
$$

Case $3^{\prime \prime} \quad$ If $g(t)=(\gamma+t) / t, \gamma>0$, then $h(t)=\gamma t+1$, and the $\mathrm{C}_{m}$-IVIM operator reduces to

$$
\begin{aligned}
& \mathrm{C}_{m^{-}}-\operatorname{IVIM}\left(\tilde{a}_{1}, \tilde{a}_{2}, \ldots, \tilde{a}_{n}\right) \\
& =\left(\left[\frac{\prod_{i=1}^{n}\left(1+\gamma \rho_{a_{(i)}}^{-}\right)^{m\left(E_{i}\right)-m\left(E_{i-1}\right)}-1}{\gamma}, \frac{\prod_{i=1}^{n}\left(1+\gamma \rho_{a_{(i)}}^{+}\right)^{m\left(E_{i}\right)-m\left(E_{i-1}\right)}-1}{\gamma}\right],\right. \\
& {\left[\frac{\gamma \prod_{i=1}^{n}\left(\sigma_{a_{(i)}^{-}}^{-}\right)^{m\left(E_{i}\right)-m\left(E_{i-1}\right)}}{\prod_{i=1}^{n}\left(\gamma+\sigma_{a_{(i)}}^{-}\right)^{m\left(E_{i}\right)-m\left(E_{i-1}\right)}-\prod_{i=1}^{n}\left(\sigma_{a_{(i)}}^{-}\right)^{m\left(E_{i}\right)-m\left(E_{i-1}\right)}},\right.} \\
& \left.\left.\frac{\gamma \prod_{i=1}^{n}\left(\sigma_{a_{(i)}}^{+}\right)^{m\left(E_{i}\right)-m\left(E_{i-1}\right)}}{\prod_{i=1}^{n}\left(\gamma+\sigma_{a_{(i)}}^{+}\right)^{m\left(E_{i}\right)-m\left(E_{i-1}\right)}-\prod_{i=1}^{n}\left(\sigma_{a_{(i)}}^{+}\right)^{m\left(E_{i}\right)-m\left(E_{i-1}\right)}}\right]\right) \text {. }
\end{aligned}
$$


Especially, if $\gamma=1$, then Eq. (35) reduces to Eq. (33); if $\gamma=2$, then Eq. (35) reduces to Eq. (34).

Generally speaking, it is not easy to determine fuzzy measure. Sometimes, it is even much more difficult than determining the weights in the weighted average method, due to the non-additive of fuzzy measures. Many scholars have devoted themselves to constructing fuzzy measures and some methods have been developed. For example, Wang and Wang (1997) used a neural network algorithm to optimize the inverse problem of synthetic evaluation to determine the Sugeno measures by the Choquet integral and statistics of given data. Combarro and Miranda (2010) studied the vertices of polytopes on the structure of the $k$-additive measures and provided an algorithm to compute the vertices descripted by the authors. How to determine the fuzzy measure is recognized as a challenging problem. Because it is not the essence of this paper, we will not elaborate it here and assign $n$ values in advance in the following examples, which can be determined by using the existing method (Wang and Wang 1997).

Next, we use a simple example adapted from (Beliakov et al. 2007) to illustrate the proposed aggregation operators:

Example 3 Consider a problem involving the evaluation of two students $X=\left\{x_{1}, x_{2}\right\}$ in a same class with respect to three subjects $E=\left\{e_{1}, e_{2}, e_{3}\right\}$ : mathematics, physics, and literature. Their marks expressed by IVIMNs are listed in Table 2.

Usually, this is done by using a weighted arithmetic mean, whose weights are interpreted as the importance degrees of different subjects. Let $g(t)=(\gamma+t) / t, h(t)=$ $\gamma t+1, \gamma>0$, then based on Eq. (18), we can easily obtain the aggregated IVIMNs by using the IVIMWA operator.

If $\gamma=1$, Eq. (18) reduces to Eq. (16), then

$\tilde{a}_{1}=([6.7460,11.9615],[0.0438,0.0577])$

$\tilde{a}_{2}=([8.7980,7.4853],[0.0450,0.0597])$.

By calculating the scores $s\left(\tilde{a}_{i}\right)$ of $\tilde{a}_{i}(i=1,2)$, we have $s\left(\tilde{a}_{1}\right)=8.9829, \quad s\left(\tilde{a}_{2}\right)=8.1151$.

Thus, $\tilde{a}_{1}>\tilde{a}_{2}$, by which we can get the ranking of the students' marks: $x_{1} \succ x_{2}$.

Similarly, we can obtain the same ranking $x_{1} \succ x_{2}$ when $\gamma=2$ and $\gamma=10$.

However, it is noticed that students who are good at mathematics are usually also good at physics and vice versa, as these

Table 2 Marks of students with IVIMNs

\begin{tabular}{llll}
\hline & Mathematics & Physics & Literature \\
\hline$x_{1}$ & $([4,6],[1 / 8,1 / 7])$ & $([3,5],[1 / 6,1 / 5])$ & $([2,3],[1 / 8,1 / 6])$ \\
$x_{2}$ & $([2,3],[1 / 5,1 / 4])$ & $([3,4],[1 / 8,1 / 6])$ & $([7,8],[1 / 9,1 / 8])$ \\
\hline
\end{tabular}

disciplines present some overlap. Thus, the evaluation by a weighted arithmetic mean would be either overestimated or underestimated for the students who are good at mathematics and physics. In what follows, we use the Sugeno measure to obtain the correlated importance of subjects. Assume that

$m(\emptyset)=0, \quad m\left(\left\{e_{1}\right\}\right)=0.3$,

$m\left(\left\{e_{2}\right\}\right)=0.2, \quad m\left(\left\{e_{3}\right\}\right)=0.4$.

By Eqs. (27) and (28), we have

$m\left(\left\{e_{1}, e_{2}\right\}\right)=0.5223, \quad m\left(\left\{e_{1}, e_{3}\right\}\right)=0.7446$,

$m\left(\left\{e_{2}, e_{3}\right\}\right)=0.6297, \quad m\left(\left\{e_{1}, e_{2}, e_{3}\right\}\right)=1$.

By calculating the scores of $\tilde{a}_{i j}(i=1,2$ and $j=1,2,3)$ :

$$
\begin{aligned}
& s\left(a_{11}\right)=36.6606, \quad s\left(a_{12}\right)=21.2132, \\
& s\left(a_{13}\right)=16.9706 \\
& s\left(a_{21}\right)=10.9545, \quad s\left(a_{22}\right)=12.0000, \\
& s\left(a_{23}\right)=63.4980
\end{aligned}
$$

we can re-rank the IVIMNs:

$\tilde{a}_{1(1)}=\tilde{a}_{11}, \quad \tilde{a}_{1(2)}=\tilde{a}_{12}, \quad \tilde{a}_{1(3)}=\tilde{a}_{13}$

$\tilde{a}_{2(1)}=\tilde{a}_{23}, \quad \tilde{a}_{2(2)}=\tilde{a}_{22}, \quad \tilde{a}_{2(3)}=\tilde{a}_{21}$

Then we can compute the weights of subjects with respect to $x_{i}(i=1,2)$, respectively, which are listed in Table 3 as follows:

Then we use the $\mathrm{C}_{m}$-IVIM operator to obtain the average value $\tilde{a}_{i}$ of the students $x_{i}(i=1,2)$ :

Case 1" Let $\gamma=1$, then based on Eq. (33), we have

$\tilde{a}_{1}=([2.7278,4.1775],[0.1331,0.1655])$,

$\tilde{a}_{2}=([3.6085,3.6060],[0.1437,0.1750])$.

Calculating the scores $s\left(\tilde{a}_{i}\right)(i=1,2)$ of $\tilde{a}_{i}(i=1,2)$ :

$s\left(\tilde{a}_{1}\right)=3.3757, \quad s\left(\tilde{a}_{2}\right)=3.6072$.

Thus, $\tilde{a}_{1}<\tilde{a}_{2}$, by which we can get the ranking of the students' marks: $x_{2} \succ x_{1}$.

Case 2" Let $\gamma=2$, then based on Eq. (34), we have

$\tilde{a}_{1}=([2.7137,4.1597],[0.1332,0.1656])$,

$\tilde{a}_{2}=([3.5568,3.5017],[0.1441,0.1755])$.

Then the scores $s\left(\tilde{a}_{i}\right)(i=1,2)$ of $\tilde{a}_{i}(i=1,2)$ can be calculated as

$s\left(\tilde{a}_{1}\right)=3.3598, \quad s\left(\tilde{a}_{2}\right)=3.5291$.

Table 3 The weights of subjects

\begin{tabular}{llll}
\hline & $m\left(E_{1}\right)$ & $m\left(E_{2}\right)-m\left(E_{1}\right)$ & $m\left(E_{3}\right)-m\left(E_{2}\right)$ \\
\hline$\tilde{a}_{1(j)}$ & 0.3000 & 0.2223 & 0.4777 \\
$\tilde{a}_{2(j)}$ & 0.4000 & 0.2297 & 0.3703 \\
\hline
\end{tabular}


Therefore, $\tilde{a}_{1}<\tilde{a}_{2}$, by which we can get the ranking of the students' marks: $x_{2} \succ x_{1}$.

Case $3^{\prime \prime}$ Let $\gamma=10$, then based on Eq. (35), we have

$\tilde{a}_{1}=([2.6989,4.1424],[0.1332,0.1657])$,

$\tilde{a}_{2}=([3.5056,3.3871],[0.1443,0.1761])$.

Then the scores $s\left(\tilde{a}_{i}\right)(i=1,2)$ of $\tilde{a}_{i}(i=1,2)$ can be calculated as

$s\left(\tilde{a}_{1}\right)=3.3437, \quad s\left(\tilde{a}_{2}\right)=3.4458$.

Thus, $\tilde{a}_{1}<\tilde{a}_{2}$, by which we can get the ranking of the students' marks: $x_{2} \succ x_{1}$.

In the above three cases, we obtain the same ranking: $x_{2} \succ x_{1}$. We can find that $x_{1}$ has advantages over $x_{2}$ in both mathematics and physics, whereas $x_{2}$ has advantages over $x_{1}$ only in literature. Even so, it does not mean that $x_{1}$ is synthetically better than $x_{2}$. The importance of mathematics and physics presents some overlap, which means that the weight of the pair $\left(e_{1}, e_{2}\right)$ is less than the sum of the weights of $e_{1}$ and $e_{2}$. Thus, after aggregating the individual IVIMNs by the $\mathrm{C}_{m}$-IVIM operator, we obtain the ranking of the students' marks: $x_{2} \succ x_{1}$, which is in accordance with the reality.

In what follows, we discuss the performance of the $\mathrm{C}_{m^{-}}$ IVIM operator according to the variations of $m\left(\left\{e_{i}\right\}\right)(j=1,2,3)$ :

Example 4 We still consider the problem in Example 3 under similar conditions but the Sugeno measure.

(I) Assume that $m\left(\left\{e_{2}\right\}\right)$ and $m\left(\left\{e_{3}\right\}\right)$ remain unchanged, while $m\left(\left\{e_{1}\right\}\right)$ decreases:

$m(\emptyset)=0, \quad m\left(\left\{e_{1}\right\}\right)=0.2$,

$m\left(\left\{e_{2}\right\}\right)=0.2, \quad m\left(\left\{e_{3}\right\}\right)=0.4$.

By Eqs. (27) and (28), we have

$m\left(\left\{e_{1}, e_{2}\right\}\right)=0.4327, \quad m\left(\left\{e_{1}, e_{3}\right\}\right)=0.6745$,

$m\left(\left\{e_{2}, e_{3}\right\}\right)=0.6745, \quad m\left(\left\{e_{1}, e_{2}, e_{3}\right\}\right)=1$.

Compared with the results in Example 3, the value of $m\left(\left\{e_{2}, e_{3}\right\}\right)$ increases which means the degree of the correlated importance becomes larger. In addition, both the values of $m\left(\left\{e_{1}, e_{2}\right\}\right)$ and $m\left(\left\{e_{1}, e_{3}\right\}\right)$ decrease which means the subject $e_{3}$ maintains the high correlated importance.

Then we can compute the weights of subjects with respect to $x_{i}(i=1,2)$, respectively, which are listed in Table 4 as follows:

Then we use the $\mathrm{C}_{m}$-IVIM operator to obtain the average value $\tilde{a}_{i}$ of the students $x_{i}(i=1,2)$ :

Case $1^{\prime \prime}$ Let $\gamma=1$, then based on Eq. (33), we have

$\tilde{a}_{1}=([2.5527,3.9164],[0.1335,0.1685])$,

$\tilde{a}_{2}=([3.4676,3.3059],[0.1445,0.1773])$.
Table 4 The weights of subjects (I)

\begin{tabular}{llll}
\hline & $m\left(E_{1}\right)$ & $m\left(E_{2}\right)-m\left(E_{1}\right)$ & $m\left(E_{3}\right)-m\left(E_{2}\right)$ \\
\hline$\tilde{a}_{1(j)}$ & 0.2000 & 0.2327 & 0.5673 \\
$\tilde{a}_{2(j)}$ & 0.4000 & 0.2745 & 0.3255 \\
\hline
\end{tabular}

Calculating the scores $s\left(\tilde{a}_{i}\right)(i=1,2)$ of $\tilde{a}_{i}(i=1,2)$ : $s\left(\tilde{a}_{1}\right)=3.1619, \quad s\left(\tilde{a}_{2}\right)=3.3858$.

Thus, $\tilde{a}_{1}<\tilde{a}_{2}$, by which we can get the ranking of the students' marks: $x_{1} \prec x_{2}$.

Case $2^{\prime \prime}$-Case $3^{\prime \prime} \quad$ Similarly, when $\gamma=2$ and $\gamma=10$, we also can have the same result: $x_{1} \prec x_{2}$, which is in accordance with Example 3.

(II) Assume that $m\left(\left\{e_{1}\right\}\right)$ and $m\left(\left\{e_{2}\right\}\right)$ remain unchanged, while $m\left(\left\{e_{3}\right\}\right)$ increases:

$m(\emptyset)=0, \quad m\left(\left\{e_{1}\right\}\right)=0.3$,

$m\left(\left\{e_{2}\right\}\right)=0.2, \quad m\left(\left\{e_{3}\right\}\right)=0.6$.

Here, the algebraic sum of the Sugeno measure of three subjects is more than one. By Eqs. (27) and (28), we have $m\left(\left\{e_{1}, e_{2}\right\}\right)=0.4823, \quad m\left(\left\{e_{1}, e_{3}\right\}\right)=0.8485$, $m\left(\left\{e_{2}, e_{3}\right\}\right)=0.7657, \quad m\left(\left\{e_{1}, e_{2}, e_{3}\right\}\right)=1$.

Then we can compute the weights of subjects with respect to $x_{i}(i=1,2)$, respectively, which are listed in Table 5 as follows:

Then we use the $\mathrm{C}_{m}$ - IVIM operator to obtain the average value $\tilde{a}_{i}$ of the students $x_{i}(i=1,2)$ :

Case 1" Let $\gamma=1$, then based on Eq. (33), we have $\tilde{a}_{1}=([2.6851,4.0942],[0.1316,0.1644])$, $\tilde{a}_{2}=([2.9594,3.3122],[0.1604,0.1973])$.

Calculating the scores $s\left(\tilde{a}_{i}\right)(i=1,2)$ of $\tilde{a}_{i}(i=1,2)$ : $s\left(\tilde{a}_{1}\right)=3.3156, \quad s\left(\tilde{a}_{2}\right)=3.1308$.

Thus, $\tilde{a}_{1}>\tilde{a}_{2}$, by which we can get the ranking of the students' marks: $x_{1} \succ x_{2}$.

Case $2^{\prime \prime}$-Case $3^{\prime \prime} \quad$ Similarly, when $\gamma=2$ and $\gamma=10$, we also can have the same result: $x_{1} \succ x_{2}$, which is contrary to the above two situations.

Table 5 The weights of subjects (II)

\begin{tabular}{llll}
\hline & $m\left(E_{1}\right)$ & $m\left(E_{2}\right)-m\left(E_{1}\right)$ & $m\left(E_{3}\right)-m\left(E_{2}\right)$ \\
\hline$\tilde{a}_{1(j)}$ & 0.3000 & 0.1823 & 0.5177 \\
$\tilde{a}_{2(j)}$ & 0.6000 & 0.1657 & 0.2343 \\
\hline
\end{tabular}


Obviously, the value of $m\left(\left\{e_{3}\right\}\right)$ increases, and both the values of $m\left(\left\{e_{1}, e_{3}\right\}\right)$ and $m\left(\left\{e_{2}, e_{3}\right\}\right)$ increase correspondingly. Thus, the correlations between $e_{3}$ and another two subjects enhance which leads to the reduction of the correlated importance of subject $e_{3}$.

\section{An approach for GDM based on interval-valued intuitionistic multiplicative preference relations}

Suppose that there are $n$ alternatives $x_{i}(i=1,2, \ldots, n)$ to be evaluated, and $t$ correlated experts $e_{k}(k=1,2, \ldots, t)$ are authorized to compare these alternatives and give their preferences with IVIMPRs, then $t$ IVIMPRs $\tilde{A}^{(k)}=$ $\left(\tilde{a}_{i j}^{(k)}\right)_{n \times n}(k=1,2, \ldots, t)$ can be constructed. To get the ranking of the alternatives, the following steps are exploited:

Step 1 Utilize the IVIMWA or IVIMOWA operator to calculate the average value $\tilde{a}_{i}^{(k)}$ of the alternative $x_{i}$ for the expert $e_{k}$ :

$$
\begin{aligned}
\tilde{a}_{i}^{(k)} & =\operatorname{IVIMWA}\left(\tilde{a}_{1 j}^{(k)}, \tilde{a}_{2 j}^{(k)}, \ldots, \tilde{a}_{n j}^{(k)}\right) \\
& =\left(\left[h^{-1}\left(\prod_{j=1}^{n}\left(h\left(\rho_{a_{i j}^{(k)}}^{-}\right)\right)^{\omega_{i}}\right), h^{-1}\left(\prod_{j=1}^{n}\left(h\left(\rho_{a_{i j}^{(k)}}^{+}\right)\right)^{\omega_{i}}\right)\right],\right. \\
& {\left.\left[g^{-1}\left(\prod_{j=1}^{n}\left(g\left(\sigma_{a_{i j}^{(k)}}^{-}\right)\right)^{\omega_{i}}\right), g^{-1}\left(\prod_{j=1}^{n}\left(g\left(\sigma_{a_{i j}^{(k)}}^{+}\right)\right)^{\omega_{i}}\right)\right]\right) }
\end{aligned}
$$

or

$$
\begin{aligned}
\tilde{a}_{i}^{(k)} & =\operatorname{IVIMOWA}\left(\tilde{a}_{1 j}^{(k)}, \tilde{a}_{2 j}^{(k)}, \ldots, \tilde{a}_{n j}^{(k)}\right) \\
& =\left(\left[h^{-1}\left(\prod_{j=1}^{n}\left(h\left(\rho_{a_{i(j)}^{(k)}}^{-}\right)\right)^{\omega_{i}}\right), h^{-1}\left(\prod_{j=1}^{n}\left(h\left(\rho_{a_{i(j)}^{+}}^{+}\right)\right)^{\omega_{i}}\right)\right],\right. \\
& {\left.\left[g^{-1}\left(\prod_{j=1}^{n}\left(g\left(\sigma_{a_{i(j)}^{-(k)}}^{-}\right)\right)^{\omega_{i}}\right), g^{-1}\left(\prod_{j=1}^{n}\left(g\left(\sigma_{a_{i(j)}^{(k)}}^{+}\right)\right)^{\omega_{i}}\right)\right]\right) . }
\end{aligned}
$$

Step 2 Use the $\mathrm{C}_{m}$-IVIM operator to obtain the average value $\tilde{a}_{i}$ of the alternative $x_{i}$ :

$$
\begin{aligned}
& \tilde{a}_{i}=\mathrm{C}_{m^{-}} \operatorname{IVIM}\left(\tilde{a}_{i}^{(1)}, \tilde{a}_{i}^{(2)}, \ldots, \tilde{a}_{i}^{(t)}\right)=\sum_{k=1}^{t}\left(m\left(E_{i}\right)-m\left(E_{i-1}\right)\right) \cdot \tilde{a}_{i}^{\operatorname{ind}(k)} \\
& =\left(\left[h^{-1}\left(\prod_{k=1}^{t}\left(h\left(\rho_{\left.a_{i}^{\text {ind }(k)}\right)}^{-}\right)\right)^{m\left(E_{i}\right)-m\left(E_{i-1}\right)}\right), h^{-1}\left(\prod_{k=1}^{t}\left(h\left(\rho_{\left.a_{i}^{\text {ind }(k)}\right)}^{+}\right)\right)^{m\left(E_{i}\right)-m\left(E_{i-1}\right)}\right)\right],\right. \\
& \left.\left[g^{-1}\left(\prod_{k=1}^{t}\left(g\left(\sigma_{a_{i}^{\text {ind }(k)}}^{-}\right)\right)^{m\left(E_{i}\right)-m\left(E_{i-1}\right)}\right), g^{-1}\left(\prod_{k=1}^{t}\left(g\left(\sigma_{a_{i}^{\text {ind }(k)}}^{+}\right)\right)^{m\left(E_{i}\right)-m\left(E_{i-1}\right)}\right)\right]\right),
\end{aligned}
$$

where $\tilde{a}_{i}^{\operatorname{ind}(k)}$ is a non-increasing permutation of $\tilde{a}_{i}^{(k)}(k=1,2, \ldots, t)$, such that $\tilde{a}_{i}^{\text {ind }(k)}$ is the $k$-th largest $\tilde{a}_{i}^{(k)}(i=1,2, \ldots, n)$.

Step 3 Calculate the score function $s\left(\tilde{a}_{i}\right)$ and the accuracy degree $r\left(\tilde{a}_{i}\right)$ of $\tilde{a}_{i}$, and obtain the ranking of the alternatives according to Definition 2.2.

Next, we use a numerical example to illustrate our developed method:

Example 5 It is well known that communication is closely related to social development. Especially, satellite communication has wide coverage area for communication, without geographical restrictions and being less susceptible to the impact of land disasters. There is a unique advantage of satellite communication in many application places, such as remote areas, mountains, islands, oceangoing vessels, and voyage aircrafts. Therefore, satellite communication not only effectively supplements the lack of other means of communication, but also has an irreplaceable role as the primary method of communication in the mass media, especially to military. In modern warfare, it is very important to quantify the quality of the communication service. Suppose that a communication joint department in China plans to design a satellite communication system. Thus, a new test methodology for the synthetic communication system is required to be investigated, in order to provide an evidence for building satellite earth stations in future. According to expeditions, there are four possible testing venues $x_{i}(i=1,2,3,4)$ to choose from (Xichang $x_{1}$, Chengdu $x_{2}$, Nanjing $x_{3}$, and Lhasa $x_{4}$ ). However, due to funds, resources, and other factors, only the most suitable one of them would be selected from. A decision-making group composed of five experts $e_{k}(k=1,2,3,4,5)$ (meteorology expert $e_{1}$, topography expert $e_{2}$, communication expert $e_{3}$, military tactical expert $e_{4}$ and military strategist $e_{5}$ ) gives the judgments with five individual IVIMPRs as follows: 


$$
\begin{aligned}
& \tilde{A}^{(1)}=\left(\begin{array}{cccc}
([1,1],[1,1]) & ([4 / 3,2],[1 / 9,1 / 7]) & ([9 / 2,5],[1 / 6,1 / 5]) & ([14 / 3,6],[1 / 8,1 / 7]) \\
([1 / 9,1 / 7],[4 / 3,2]) & ([1,1],[1,1]) & ([16 / 3,6],[1 / 8,1 / 6]) & ([9 / 2,5],[1 / 6,1 / 6]) \\
([1 / 6,1 / 5],[9 / 2,5]) & ([1 / 8,1 / 6],[16 / 3,6]) & ([1,1],[1,1]) & ([8 / 3,5],[1 / 7,1 / 6]) \\
([1 / 8,1 / 7],[14 / 3,6]) & ([1 / 6,1 / 6],[9 / 2,5]) & ([1 / 7,1 / 6],[8 / 3,5]) & ([1,1],[1,1])
\end{array}\right)_{4 \times 4} \\
& \tilde{A}^{(2)}=\left(\begin{array}{cccc}
([1,1],[1,1]) & ([5,21 / 3],[1 / 9,1 / 7]) & ([3,4],[1 / 6,1 / 4]) & ([2,3],[1 / 8,1 / 4]) \\
([1 / 9,1 / 7],[5,21 / 3]) & ([1,1],[1,1]) & ([10 / 3,5],[1 / 6,1 / 5]) & ([7 / 2,4],[1 / 6,1 / 5]) \\
([1 / 6,1 / 4],[3,4]) & ([1 / 6,1 / 5],[10 / 3,5]) & ([1,1],[1,1]) & ([3 / 2,2],[1 / 6,1 / 3]) \\
([1 / 8,1 / 4],[2,3]) & ([1 / 6,1 / 5],[7 / 2,4]) & ([1 / 6,1 / 3],[3 / 2,2]) & ([1,1],[1,1])
\end{array}\right)_{4 \times 4} \\
& \tilde{A}^{(3)}=\left(\begin{array}{cccc}
([1,1],[1,1]) & ([3 / 4,4 / 3],[1 / 3,1 / 2]) & ([5 / 2,3],[1 / 5,1 / 3]) & ([2,3],[2 / 9,1 / 4]) \\
([1 / 3,1 / 2],[3 / 4,4 / 3]) & ([1,1],[1,1]) & ([3,5],[1 / 9,1 / 6]) & ([8 / 3,4],[1 / 6,1 / 5]) \\
([1 / 5,1 / 3],[5 / 2,3]) & ([1 / 9,1 / 6],[3,5]) & ([1,1],[1,1]) & ([4,5],[1 / 8,1 / 6]) \\
([2 / 9,1 / 4],[2,3]) & ([1 / 6,1 / 5],[8 / 3,4]) & ([1 / 8,1 / 6],[4,5]) & ([1,1],[1,1])
\end{array}\right)_{4 \times 4} \\
& \tilde{A}^{(4)}=\left(\begin{array}{cccc}
([1,1],[1,1]) & ([5 / 2,3],[1 / 5,1 / 4]) & ([5 / 3,3],[1 / 5,1 / 3]) & ([2 / 3,3],[2 / 9,1 / 4]) \\
([1 / 5,1 / 4],[5 / 2,3]) & ([1,1],[1,1]) & ([1 / 6,1 / 2],[1 / 3,1 / 2]) & ([2,4],[1 / 6,1 / 5]) \\
([1 / 5,1 / 3],[5 / 3,3]) & ([1 / 3,1 / 2],[1 / 6,1 / 2]) & ([1,1],[1,1]) & ([7 / 2,4],[1 / 7,1 / 5]) \\
([2 / 9,1 / 4],[2 / 3,3]) & ([1 / 6,1 / 5],[2,4]) & ([1 / 7,1 / 5],[7 / 2,4]) & ([1,1],[1,1])
\end{array}\right)_{4 \times 4} \\
& \tilde{A}^{(5)}=\left(\begin{array}{cccc}
([1,1],[1,1]) & ([4,6],[1 / 9,1 / 8]) & ([2 / 3,3],[1 / 8,1 / 6]) & ([3 / 2,2],[2 / 9,1 / 4]) \\
([1 / 9,1 / 8],[4,6]) & ([1,1],[1,1]) & ([1 / 3,1 / 2],[2 / 5,1 / 2]) & ([3,4],[1 / 9,1 / 6]) \\
([1 / 8,1 / 6],[2 / 3,3]) & ([2 / 5,1 / 2],[1 / 3,1 / 2]) & ([1,1],[1,1]) & ([2,8 / 3],[2 / 9,2 / 7]) \\
([2 / 9,1 / 4],[3 / 2,2]) & ([1 / 9,1 / 6],[3,4]) & ([2 / 9,2 / 7],[2,8 / 3]) & ([1,1],[1,1])
\end{array}\right)_{4 \times 4}
\end{aligned}
$$

Suppose that the weight vector of venues is $\tilde{a}_{3}^{(1)}=([0.7614,1.0245],[0.8367,0.9057])$, $\omega=(1 / 4,1 / 4,1 / 4,1 / 4)^{T}$.

Case I Let $\gamma=1$, in order to obtain the ranking of the alternative, we give the following steps:

Step 1 Due to $\omega_{i}=1 / 4$ (for all $i$ ) in this example, we are free to choose the IVIMWA or IVIMOWA operator. We here take the IVIMWA operator for example, Eq. (36) is equal to

$$
\begin{aligned}
& \tilde{a}_{4}^{(1)}=([0.3161,0.3281],[2.3733,2.8257]), \\
& \tilde{a}_{1}^{(2)}=([2.4641,3.2295],[0.2017,0.2880]), \\
& \tilde{a}_{2}^{(2)}=([1.5657,1.8776],[0.4361,0.4971]), \\
& \tilde{a}_{3}^{(2)}=([0.6152,0.7321],[0.8200,1.1612]),
\end{aligned}
$$

$$
\begin{aligned}
& \tilde{a}_{i}^{(k)}=\operatorname{IVIMWA}\left(\tilde{a}_{1 j}^{(k)}, \tilde{a}_{2 j}^{(k)}, \ldots, \tilde{a}_{n j}^{(k)}\right) \\
& =\left(\left[\prod_{j=1}^{n}\left(1+\rho_{a_{i j}^{(k)}}^{-}\right)^{\omega_{i}}-1, \prod_{j=1}^{n}\left(1+\rho_{a_{i j}^{(k)}}^{+}\right)^{\omega_{i}}-1\right],\left[\frac{\prod_{j=1}^{n}\left(\sigma_{a_{i j}^{(k)}}^{-}\right)^{\omega_{i}}}{\prod_{j=1}^{n}\left(1+\sigma_{a_{i j}^{(k)}}^{-}\right)^{\omega_{i}}-\prod_{j=1}^{n}\left(\sigma_{a_{i j}^{(k)}}^{-}\right)^{\omega_{i}}}, \frac{\prod_{j=1}^{n}\left(\sigma_{a_{i j}^{(k)}}^{+}\right)^{\omega_{i}}}{\left.\prod_{j=1}^{n}\left(1+\sigma_{a_{i j}^{(k)}}^{+}\right)^{\omega_{i}}-\prod_{j=1}^{n}\left(\sigma_{a_{i j}^{(k)}}^{+}\right)^{\omega_{i}}\right]}\right] .\right.
\end{aligned}
$$

Then the average values $\tilde{a}_{i}^{(k)}(i=1,2,3,4)$ of the alternatives $x_{i}(i=1,2,3,4)$ for the experts $e_{k} \quad(k=$ $1,2,3,4,5)$ are

$\tilde{a}_{1}^{(1)}=([2.4728,2.9843],[0.2017,0.2345])$,

$\tilde{a}_{2}^{(1)}=([1.9662,2.1302],[0.3505,0.4029])$, $\tilde{a}_{4}^{(2)}=([0.3229,0.4142],[1.6883,2.0188])$,

$\tilde{a}_{1}^{(3)}=([1.4621,1.9396],[0.3299,0.4329])$,

$\tilde{a}_{2}^{(3)}=([1.5008,2.0801],[0.3076,0.4029])$,

$\tilde{a}_{3}^{(3)}=([0.9109,1.0786],[0.7104,0.8507])$, 
$\tilde{a}_{4}^{(3)}=([0.3384,0.3678],[1.9728,2.4142])$,

$\tilde{a}_{1}^{(4)}=([1.3617,2.3636],[0.2889,0.3622])$,

$\tilde{a}_{2}^{(4)}=([0.7024,1.0809],[0.5062,0.6127])$,

$\tilde{a}_{3}^{(4)}=([0.9480,1.1147],[0.3761,0.6127])$,

$\tilde{a}_{4}^{(4)}=([0.3436,0.3774],[1.3120,2.3325])$,

$\tilde{a}_{1}^{(5)}=([1.5407,2.6002],[0.2170,0.2494])$,

$\tilde{a}_{2}^{(5)}=([0.8554,1.0268],[0.4858,0.6076])$,

$\tilde{a}_{3}^{(5)}=([0.7533,0.8927],[0.4467,0.6899])$,

$\tilde{a}_{4}^{(5)}=([0.3498,0.3916],[1.6478,1.9728])$.

Step 2 Assume that the decision makers have correlations with each other and

$m(\emptyset)=0, \quad m\left(\left\{e_{1}\right\}\right)=0.3, \quad m\left(\left\{e_{2}\right\}\right)=0.2$,

$m\left(\left\{e_{3}\right\}\right)=0.4, \quad m\left(\left\{e_{4}\right\}\right)=0.5, \quad m\left(\left\{e_{5}\right\}\right)=0.3$.

By Eqs. (27) and (28), we have

$m\left(\left\{e_{1}, e_{2}\right\}\right)=0.4518, \quad m\left(\left\{e_{1}, e_{3}\right\}\right)=0.6035$,

$m\left(\left\{e_{4}, e_{5}\right\}\right)=0.6794, \quad m\left(\left\{e_{1}, e_{2}, e_{3}\right\}\right)=0.7065$,

$m\left(\left\{e_{1}, e_{2}, e_{5}\right\}\right)=0.6428, \quad m\left(\left\{e_{2}, e_{4}, e_{5}\right\}\right)=0.7702$,

$m\left(\left\{e_{3}, e_{4}, e_{5}\right\}\right)=0.8609, \quad m\left(\left\{e_{1}, e_{2}, e_{3}, e_{5}\right\}\right)=0.8361$,

$m\left(\left\{e_{1}, e_{2}, e_{4}, e_{5}\right\}\right)=0.8844, \quad m\left(\left\{e_{1}, e_{3}, e_{4}, e_{5}\right\}\right)=0.9533$,

$m\left(\left\{e_{2}, e_{3}, e_{4}, e_{5}\right\}\right)=0.9225, \quad m\left(\left\{e_{1}, e_{2}, e_{3}, e_{4}, e_{5}\right\}\right)=1$.

Moreover, based on Definition 2.2, we obtain

$\tilde{a}_{1}^{\operatorname{ind}(1)}=\tilde{a}_{1}^{(1)}, \quad \tilde{a}_{1}^{\operatorname{ind}(2)}=\tilde{a}_{1}^{(2)}, \quad \tilde{a}_{1}^{\operatorname{ind}(3)}=\tilde{a}_{2}^{(5)}$,

$\tilde{a}_{1}^{\operatorname{ind}(4)}=\tilde{a}_{1}^{(4)}, \quad \tilde{a}_{1}^{\operatorname{ind}(5)}=\tilde{a}_{1}^{(3)}$,

$\tilde{a}_{2}^{\operatorname{ind}(1)}=\tilde{a}_{2}^{(1)}, \quad \tilde{a}_{2}^{\operatorname{ind}(2)}=\tilde{a}_{2}^{(3)}, \quad \tilde{a}_{2}^{\operatorname{ind}(3)}=\tilde{a}_{1}^{(2)}$,

$\tilde{a}_{2}^{\operatorname{ind}(4)}=\tilde{a}_{3}^{(5)}, \quad \tilde{a}_{2}^{\operatorname{ind}(5)}=\tilde{a}_{2}^{(4)}$,

$\tilde{a}_{3}^{\operatorname{ind}(1)}=\tilde{a}_{3}^{(4)}, \quad \tilde{a}_{3}^{\operatorname{ind}(2)}=\tilde{a}_{3}^{(5)}, \quad \tilde{a}_{3}^{\operatorname{ind}(3)}=\tilde{a}_{3}^{(3)}$,

$\tilde{a}_{3}^{\operatorname{ind}(4)}=\tilde{a}_{2}^{(1)}, \quad \tilde{a}_{3}^{\operatorname{ind}(5)}=\tilde{a}_{3}^{(2)}$,

$\tilde{a}_{4}^{\operatorname{ind}(1)}=\tilde{a}_{4}^{(4)}, \quad \tilde{a}_{4}^{\operatorname{ind}(2)}=\tilde{a}_{4}^{(5)}, \quad \tilde{a}_{4}^{\operatorname{ind}(3)}=\tilde{a}_{4}^{(2)}$,

$\tilde{a}_{4}^{\operatorname{ind}(4)}=\tilde{a}_{4}^{(3)}, \quad \tilde{a}_{4}^{\operatorname{ind}(5)}=\tilde{a}_{4}^{(1)}$.

Then we can calculate the values of $m\left(E_{i}\right)-m\left(E_{i-1}\right)$ in the $\mathrm{C}_{m}$-IVIM operator (Eq. (38)) respectively, which are listed in Table 6 as follows:

We utilize the $\mathrm{C}_{m}$-IVIM operator (Eq. (38)) to obtain the average value $\tilde{a}_{i}$ of $x_{i}(i=1,2,3,4)$ : $\tilde{a}_{1}=([1.8633,2.6546],[0.2353,0.2903])$,

$\tilde{a}_{2}=([1.3839,1.7299],[0.3797,0.4625])$,

$\tilde{a}_{3}=([0.8708,1.0392],[0.4781,0.7046])$,

$\tilde{a}_{4}=([0.3419,0.3824],[1.6635,2.1507])$.

Step 3 Calculate the scores $s\left(\tilde{a}_{i}\right)(i=1,2,3,4)$ of $\tilde{a}_{i}(i=1,2,3,4)$ :

$s\left(\tilde{a}_{1}\right)=8.5097, \quad s\left(\tilde{a}_{2}\right)=3.6923$,

$s\left(\tilde{a}_{3}\right)=1.6390, \quad s\left(\tilde{a}_{4}\right)=0.1912$.

Thus, $\tilde{a}_{1}>\tilde{a}_{2}>\tilde{a}_{3}>\tilde{a}_{4}$, by which we can get the ranking of the alternatives: $x_{1} \succ x_{2} \succ x_{3} \succ x_{4}$.

Case II-Case III Let $\gamma=2$ and $\gamma=10$. Similarly, we can compute the average value $\tilde{a}_{i}$ of $x_{i}(i=1,2,3,4)$ and the scores $s\left(\tilde{a}_{i}\right)$ of $\tilde{a}_{i}(i=1,2,3,4) .$. For comparison, the results are shown in Table 7 .

In the cases above, we obtain the same result: Xichang is the most suitable venue for building the satellite earth station. This indicates that the approach proposed offers good stability.

Next, we select the GDM method developed by $\mathrm{Yu}$ (2015a, b) to aggregate the given interval-valued intuitionistic multiplicative information and compare the results with our approach. In Yu (2015a, b), the correlations of inputs have not been taken into account in the GDM method. Although some aggregation operators for the correlated inputs have been given, $2^{n}$ values of fuzzy measures should be known in advance which is huge in quantity and not easy to realize. Thus, they are not suitable to solve the GDM problem and the correlations of inputs are ignored in GDM. While only $n$ values of fuzzy measures need to be determined in this paper, which is more suitable for GDM. Then instead of the $\mathrm{C}_{m}$-IVIM operator in Step 2, we use the IVIMWA operator to obtain the average value $\tilde{a}_{i}$ of the alternative $x_{i}$. In order to maintain unity, here we let the weights of five experts be $(3 / 17,2 / 17,4 / 17,5 / 17,3 / 17)^{T}$. The results in different cases are shown in Table 8 under other invariable conditions.

From Table 8, we find that the most suitable venue for building the satellite earth station is still Xichang. But different ranking results are obtained without considering correlations of the given interval-valued intuitionistic multiplicative information. In practical applications, designing a good alternative is also critical. Take this venue-selection problem as an example, if we use the GDM approach proposed in this paper, we should take $x_{2}$ as an 
Table $6 m\left(E_{i}\right)-m\left(E_{i-1}\right)$ in Eq. (38)

Table 7 Average value $\tilde{a}_{i}$ of $x_{i}$ and the scores $s\left(\tilde{a}_{i}\right)$ of $\tilde{a}_{i}$ using the $\mathrm{C}_{m}$-IVIM operator $(i=1,2,3,4)$

\begin{tabular}{llllll}
\hline & $m\left(E_{1}\right)$ & $m\left(E_{2}\right)-m\left(E_{1}\right)$ & $m\left(E_{3}\right)-m\left(E_{2}\right)$ & $m\left(E_{4}\right)-m\left(E_{3}\right)$ & $m\left(E_{5}\right)-m\left(E_{4}\right)$ \\
\hline$\tilde{a}_{1}$ & 0.3000 & 0.1518 & 0.1910 & 0.2416 & 0.1156 \\
$\tilde{a}_{2}$ & 0.3000 & 0.3035 & 0.1030 & 0.1296 & 0.1639 \\
$\tilde{a}_{3}$ & 0.5000 & 0.1794 & 0.1815 & 0.0924 & 0.0467 \\
$\tilde{a}_{4}$ & 0.5000 & 0.1794 & 0.0908 & 0.1523 & 0.0775 \\
\hline
\end{tabular}

\begin{tabular}{llll}
\hline$\gamma$ & Average values & Scores & Ranking \\
\hline Case I & $\tilde{a}_{1}=([1.8633,2.6546],[0.2353,0.2903])$ & $s\left(\tilde{a}_{1}\right)=8.5097$ & $x_{1} \succ x_{2} \succ x_{3} \succ x_{4}$ \\
$\gamma=1$ & $\tilde{a}_{2}=([1.3839,1.7299],[0.3797,0.4625])$ & $s\left(\tilde{a}_{2}\right)=3.6923$ & \\
& $\tilde{a}_{3}=([0.8708,1.0392],[0.4781,0.7046])$ & $s\left(\tilde{a}_{3}\right)=1.6390$ & \\
& $\tilde{a}_{4}=([0.3419,0.3824],[1.6635,2.1507])$ & $s\left(\tilde{a}_{4}\right)=0.1912$ & \\
Case II & $\tilde{a}_{1}=([1.8095,2.5935],[0.2427,0.2982])$ & $s\left(\tilde{a}_{1}\right)=8.0526$ & $x_{1} \succ x_{2} \succ x_{3} \succ x_{4}$ \\
$\gamma=2$ & $\tilde{a}_{2}=([1.2661,1.5845],[0.4051,0.4952])$ & $s\left(\tilde{a}_{2}\right)=3.1620$ & \\
& $\tilde{a}_{3}=([0.7935,0.9561],[0.5167,0.7621])$ & $s\left(\tilde{a}_{3}\right)=1.3888$ & \\
Case III & $\tilde{a}_{4}=([0.3233,0.3665],[1.6996,2.2119])$ & $s\left(\tilde{a}_{4}\right)=0.1775$ & \\
$\gamma=10$ & $\tilde{a}_{1}=([1.7457,2.5242],[0.2516,0.3077])$ & $s\left(\tilde{a}_{1}\right)=7.5443$ & $x_{1} \succ x_{2} \succ x_{3} \succ x_{4}$ \\
& $\tilde{a}_{2}=([1.0776,1.3625],[0.4429,0.5492])$ & $s\left(\tilde{a}_{2}\right)=2.4569$ & \\
& $\tilde{a}_{3}=([0.6681,0.8351],[0.5831,0.8541])$ & $s\left(\tilde{a}_{3}\right)=1.0585$ & \\
& $\tilde{a}_{4}=([0.2942,0.3256],[1.7703,2.3412])$ & $s\left(\tilde{a}_{4}\right)=0.1520$ & \\
\hline
\end{tabular}

Table 8 Average value $\tilde{a}_{i}$ of $x_{i}$ and the scores $s\left(\tilde{a}_{i}\right)$ of $\tilde{a}_{i}$ using the IVIMWA operator $(i=1,2,3,4)$

\begin{tabular}{llll}
\hline$\gamma$ & Average values & Scores & Ranking \\
\hline Case I & $\tilde{a}_{1}=([1.4947,2.0920],[0.3088,0.3815])$ & $s\left(\tilde{a}_{1}\right)=5.1515$ & $x_{1} \succ x_{3} \succ x_{2} \succ x_{4}$ \\
$\gamma=1$ & $\tilde{a}_{2}=([0.6192,0.7511],[0.9320,1.0886])$ & $s\left(\tilde{a}_{2}\right)=0.6771$ & \\
& $\tilde{a}_{3}=([0.9885,1.2116],[0.4639,0.6261])$ & $s\left(\tilde{a}_{3}\right)=2.0307$ & \\
& $\tilde{a}_{4}=([0.5336,0.5923],[1.0545,1.4138])$ & $s\left(\tilde{a}_{4}\right)=0.4604$ & \\
Case II & $\tilde{a}_{1}=([1.3981,1.9547],[0.3351,0.4111])$ & $s\left(\tilde{a}_{1}\right)=4.4538$ & $x_{1} \succ x_{3} \succ x_{4} \succ x_{2}$ \\
$\gamma=2$ & $\tilde{a}_{2}=([0.5117,0.6144],[1.1466,1.3289])$ & $s\left(\tilde{a}_{2}\right)=0.4543$ & \\
& $\tilde{a}_{3}=([0.9324,1.1494],[0.4828,0.6586])$ & $s\left(\tilde{a}_{3}\right)=1.8360$ & \\
Case III & $\tilde{a}_{4}=([0.5323,0.6015],[0.9924,1.3608])$ & $s\left(\tilde{a}_{4}\right)=0.4869$ & \\
$\gamma=10$ & $\tilde{a}_{1}=([1.1857,1.6525],[0.4038,0.4908])$ & $s\left(\tilde{a}_{1}\right)=3.1440$ & \\
& $\tilde{a}_{2}=([0.3034,0.3611],[1.9654,2.2582])$ & $s\left(\tilde{a}_{2}\right)=0.1571$ & \\
& $\tilde{a}_{3}=([0.8921,1.1429],[0.4625,0.6547])$ & $s\left(\tilde{a}_{3}\right)=1.8350$ & \\
& $\tilde{a}_{4}=([0.6127,0.7337],[0.7129,1.0448])$ & $s\left(\tilde{a}_{4}\right)=0.7768$ & \\
& & &
\end{tabular}

alternative. While, if we choose the GDM method in (Yu $2015 \mathrm{a}$, b) without considering the interrelationships of inputs, we shall make $x_{3}$ an alternative to $x_{1}$. Thus, to capture the interrelationships among the aggregated information is important in the GDM problems.

\section{Concluding Remarks}

In this paper, we have introduced some interval-valued intuitionistic multiplicative aggregation operators and presented an approach for GDM where the correlated decision makers provide their preference information over alternatives by using IVIMPRs. To aggregate the interval-valued intuitionistic multiplicative information, several aggregation operators have been developed and their properties also have been discussed and proven. The contributions of this paper are as follows: (1) the IVIMWA and IVIMOWA operators based on the pseudo-multiplication can be utilized to aggregate a collection of IVIMNs which is more general. Based on different aggregation operations, several specific forms of the IVIMWA and IVIMOWA operators can be obtained. (2) The $\mathrm{C}_{m}$-IVIM operator, based on the Choquet integral and Sugeno measure, can be used for aggregating the 
correlated IVIMNs whose weights can be easily calculated. (3) The proposed approach for GDM can deal with the situations where the preference information is constructed by IVIMPRs and there exist the correlations or the connections among the decision makers. Of course, the proposed approach has its limitations: (1) the formulas of the aggregation operators proposed in this paper seem to be a little complex. (2) How to determine the fuzzy measure has not been discussed deeply, but is important and cannot be avoided with the deepening of the research. (3) Other families of fuzzy measures still need to be investigated, such as $k$ additive fuzzy measures, the $p$-symmetric measures, the decomposable measures and so on. Their advantages and disadvantages are also worth studying. In the future, we will deepen our research and apply our approach to solve more practical GDM problems with IVIMPRs.

\section{References}

Atanassov K, Gargov G (1989) Interval valued intuitionistic fuzzy sets. Fuzzy Sets Syst 31:343-349

Beliakov G, Pradera A, Calvo T (2007) Aggregation functions: a guide for practitioners, vol 221. Springer, Berlin

Benvenuti P, Mesiar R (2004) Pseudo-arithmetical operations as a basis for the general measure and integration theory. Inf Sci Inform Comput Sci Int J 160:1-11

Choquet G (1953) Theory of capacities. Annales de l'Institut Fourier (Crenoble) 5:131-295

Combarro EF, Miranda P (2010) On the structure of the k-additive fuzzy measures. Fuzzy Set Syst 161:2314-2327

Conde E, Pérez MPR (2010) A linear optimization problem to derive relative weights using an interval judgement matrix. Eur J Oper Res 201:537-544

Fang L, Zhang WG, Wang ZX (2012) A goal programming model for incomplete interval multiplicative preference relations and its application in group decision-making. Eur $\mathbf{J}$ Oper Res 218:747-754

Grabisch M (1996) The application of fuzzy integrals in multicriteria decision making. Eur J Oper Res 89:445-456

ITU-R Recommendation ITU-R RPN.618-4 (1996)

Jiang Y, Xu ZS, Yu XH (2013) Compatibility measures and consensus models for group decision making with intuitionistic multiplicative preference relations. Appl Soft Comput 13:2075-2086

Jiang Y, Xu ZS, Xu JP (2014) Interval-valued intuitionistic multiplicative set. Int $\mathrm{J}$ Uncertain Fuzziness Knowl Based Syst 22:385-406

Jiang Y, Xu ZS, Yu XH (2015a) Group decision making based on incomplete intuitionistic multiplicative preference relations. Inf Sci 295:33-52

Jiang Y, Xu ZS, Gao M (2015b) Methods for ranking intuitionistic multiplicative numbers by distance measures in decision making. Comput Ind Eng 88:100-109

Jiang Y, Xu ZS, Shu YH (2016) Distance measures between intuitionistic multiplicative sets and their application in selection of satellite earth stations. Syst Eng Theory Pract 36:3210-3219

Li CQ, Zhao H, Xu ZS (2017) Kernel C-means clustering algorithms for hesitant fuzzy information in decision making. Int J Fuzzy Syst 1-14. doi:10.1007/s40815-017-0304-3
Liang DC, Xu ZS, Liu D (2017) Three-way decisions based on decision-theoretic rough sets with dual hesitant fuzzy information. Inf Sci 396:127-143

Liu F (2009) Acceptable consistency analysis of interval reciprocal comparison matrices. Fuzzy Sets Syst 160:2686-2700

Orlovsky SA (1978) Decision-making with a fuzzy preference relation. Fuzzy Sets Syst 1:155-167

Pap E, Ralević N (1998) Pseudo-Laplace transform. Nonlinear Anal 33:533-550

Pinar AJ, Anderson DT, Havens TC, Zare A, Adeyeba T (2017) Measures of the Shapley index for learning lower complexity fuzzy integrals. Granul Comput 6:1-17

Qin J (2017) Interval type-2 fuzzy Hamy mean operators and their application in multiple criteria decision making. Granul Comput $7: 1-21$

Ren PJ, Xu ZS, Lei Q (2016) Simplified interval-valued intuitionistic fuzzy sets with intuitionistic fuzzy numbers. J Intell Fuzzy Syst 30:2871-2882

Ren PJ, Xu ZS, Hao ZN (2017) Hesitant fuzzy thermodynamic method for emergency decision making based on prospect theory. IEEE Trans Cybern 99:1-13

Saaty TL (1977) A scaling method for priorities in hierarchy structures. J Math Psychol 15:234-281

Saaty TL (1980) The analytic hierarchy process. McGraw-Hill, New York

Sanchez MA, Castro JR, Castillo O, Mendoza O, Rodriguez-Diaz A, Melin P (2017) Fuzzy higher type information granules from an uncertainty measurement. Granul Comput 2:95-103

Sugeno M (1974) Theory of fuzzy integrals and applications. Tokyo Institute of Technology, Tokyo, Japan

Sugeno M, Murofushi T (1987) Pseudo-additive measures and integrals. J Math Anal Appl 122:197-222

Wang YM, Elhag TMS (2007) A goal programming method for obtaining interval weights from an interval comparison matrix. Eur J Oper Res 177:458-471

Wang J, Wang Z (1997) Using neural networks to determine Sugeno measures by statistics. Neural Netw 10:183-195

Xia MM, Xu ZS, Liao HC (2013) Preference relations based on intuitionistic multiplicative information. IEEE Trans Fuzzy Syst 21:113-133

Xu ZS (2007) Intuitionistic preference relations and their application in group decision making. Inf Sci 177:2363-2379

$\mathrm{Xu}$ ZS (2010) A method based on distance measure for intervalvalued intuitionistic fuzzy group decision making. Inf Sci 180:181-190

Xu ZS, Chen J (2008) Some models for deriving the priority weights from interval fuzzy preference relations. Eur J Oper Res 184:266-280

Yager RR (1988) On ordered weighted averaging aggregation operators in multicriteria decision making. IEEE Trans Fuzzy Syst Man Cybern 18:183-190

Yager RR (2004) Choquet aggregation using order inducing variables. Int J Uncertain Fuzziness Knowl Based Syst 12:69-88

Yu DJ (2015a) Group decision making under interval-valued multiplicative intuitionistic fuzzy environment based on Archimedean t-conorm and t-norm. Int J Intell Syst 30:590-616

$\mathrm{Yu}$ DJ (2015b) A scientometrics review on aggregation operator research. Scientometrics 105:115-133

Yu DJ, Shi SS (2015) Researching the development of Atanassov intuitionistic fuzzy set: using a citation network analysis. Appl Soft Comput 32:189-198

Yu DJ, Merigó JM, Zhou LG (2013) Interval-valued multiplicative intuitionistic fuzzy preference relations. Int J Fuzzy Syst 15:412-422

Zadeh LA (1975) The concept of a linguistic variable and its application to approximate reasoning-I. Inf Sci 8:199-249 